\title{
Number of Spanning Trees of Different Products of Complete and Complete Bipartite Graphs
}

\author{
S. N. Daoud ${ }^{1,2}$ \\ ${ }^{1}$ Department of Mathematics, Faculty of Science, Menoufia University, Shebin Elkom 32511, Egypt \\ ${ }^{2}$ Department of Mathematics, Faculty of Science, Taibah University, Al Madinah 41411, Saudi Arabia
}

Correspondence should be addressed to S. N. Daoud; sa_na_daoud@yahoo.com

Received 28 December 2013; Revised 31 May 2014; Accepted 31 May 2014; Published 2 July 2014

Academic Editor: Efstratios Tzirtzilakis

Copyright @ 2014 S. N. Daoud. This is an open access article distributed under the Creative Commons Attribution License, which permits unrestricted use, distribution, and reproduction in any medium, provided the original work is properly cited.

Spanning trees have been found to be structures of paramount importance in both theoretical and practical problems. In this paper we derive new formulas for the complexity, number of spanning trees, of some products of complete and complete bipartite graphs such as Cartesian product, normal product, composition product, tensor product, symmetric product, and strong sum, using linear algebra and matrix theory techniques.

\section{Introduction}

The number of spanning trees of a graph is an important, well-studied quantity in graph theory and appears in a number of applications. The most notable application fields are network reliability [1-4], enumerating certain chemical isomers [5], and counting of the Eulerian circuits in a graph [6]. Every connected graph has a spanning tree. A spanning tree of a graph $G$ is a tree that (i) is a subgraph of $G$ (i.e., that includes only edges from $G$ ) and (ii) includes every vertex of $G$. The most classical interest concerning a spanning tree is the number of spanning trees, also called the complexity of the graph $G$ and denoted by $\tau(G)$. Kirchhoff [7] gave a formula for determining it, which is known as the matrix tree theorem. The spanning trees of a graph $G$ are the value of any cofactor of the matrix $D(G)-A(G)$, where $D(G)$ is the degree matrix (the $i$ th diagonal entry is equal to the degree of the $i$ th vertex and the other entry is equal to zero) and $A(G)$ is the adjacency matrix of $G$ (the entry $(i, j)$ is equal to the number of edges between $i$ th vertex and $j$ th vertex), respectively. This topic is still much studied, in particular, in explicit formulas of the number of spanning trees of some special classes.
That for complete graphs is most famous among such classes; the number of spanning trees of $K_{n}$ is $n^{n-2}$, called Cayley's formula [8]. Several proofs of Cayley's formula are known, and the most famous one is due to Prüfer [9]. The explicit formulas of the number of spanning trees are known for other classes than complete graphs: complete bipartite graphs [1013], regular graphs [14], circulant graphs [15-19], pyramid graphs [20], and so on.

Now we introduce the following Lemma which describes a way to calculate the number of spanning trees by an extension of Kirchhoff formula.

Lemma 1 (see [21]). Let $G$ be a graph with $n$ vertices. Then

$$
\tau(G)=\frac{1}{n^{2}} \operatorname{det}(n I-\bar{D}+\bar{A})
$$

where $\bar{A}, \bar{D}$ are the adjacency and degree matrices of $\bar{G}$, the complement of $G$, respectively, and $I$ is the $n \times n$ unit matrix. 
Lemma 2. Let $A_{n}(x)$ be $n \times n$ matrix, $x \geq 2$ such that

$$
A_{n}(x)=\left(\begin{array}{cccccc}
x & 1 & \cdots & \cdots & \cdots & 1 \\
1 & x & \ddots & \ddots & \ddots & \vdots \\
\vdots & \ddots & \ddots & \ddots & \ddots & \vdots \\
\vdots & \ddots & \ddots & \ddots & \ddots & \vdots \\
\vdots & \ddots & \ddots & \ddots & x & 1 \\
1 & \cdots & \cdots & \cdots & 1 & x
\end{array}\right) .
$$

Then

$$
\operatorname{det}\left(A_{n}(x)\right)=(x+n-1)(x-1)^{n-1} \text {. }
$$

Proof. From the definition of the circulant determinants, we have

$$
\begin{aligned}
& \operatorname{det}\left(A_{n}(x)\right)=\operatorname{det}\left(\begin{array}{cccccc}
x & 1 & \cdots & \cdots & \cdots & 1 \\
1 & x & \ddots & \ddots & \ddots & \vdots \\
\vdots & \ddots & \ddots & \ddots & \ddots & \vdots \\
\vdots & \ddots & \ddots & \ddots & \ddots & \vdots \\
\vdots & \ddots & \ddots & \ddots & x & 1 \\
1 & \cdots & \cdots & \cdots & 1 & x
\end{array}\right) \\
& =\prod_{j=1}^{n}\left(x+\omega_{j}+\omega_{j}^{2}+\omega_{j}^{3}+\cdots+\omega_{j}^{n-1}\right) \\
& =(x+1+1+\cdots+1) \\
& \times \prod_{j=1, \omega_{j} \neq 1}^{n}(x+\underbrace{\omega_{j}+\omega_{j}^{2}+\omega_{j}^{3}+\cdots+\omega_{j}^{n-1}}_{=-1}) \\
& =(x+n-1) \times(x-1)^{n-1} .
\end{aligned}
$$

Then

$$
\operatorname{det} \mathbb{F}=[\operatorname{det}(A-B)]^{k-1} \operatorname{det}[A+(k-1) B] .
$$

Lemma 4 (see [22]). Let $A \in F^{n \times n}, B \in F^{n \times m}, C \in F^{m \times n}$, and $D \in F^{m \times m}$. Assume that $A$ and $D$ are nonsingular matrices. Then:

$$
\begin{aligned}
\operatorname{det}\left(\begin{array}{ll}
A & B \\
C & D
\end{array}\right) & =(-1)^{n m} \operatorname{det}\left(A-B D^{-1} C\right) \operatorname{det} D \\
& =(-1)^{n m} \operatorname{det} A \operatorname{det}\left(D-C A^{-1} B\right) .
\end{aligned}
$$

Formulas in Lemmas 2, 3, and 4 give some sort of symmetry in some matrices which facilitates our calculation of determinants.

\section{Number of Spanning Trees of Cartesian Product of Graphs}

The Cartesian product, $G_{1} \times G_{2}$, of two graphs $G_{1}$ and $G_{2}$ is the simple graph with vertex set $V\left(G_{1} \times G_{2}\right)=V_{1} \times V_{2}$ and edge set $E\left(G_{1} \times G_{2}\right)=\left[\left(E_{1} \times V_{2}\right) \cup\left(V_{1} \times E_{2}\right)\right]$ such that two vertices $\left(u_{1}, u_{2}\right)$ and $\left(v_{1}, v_{2}\right)$ are adjacent in $G_{1} \times G_{2}$ if and only if either $u_{1}=v_{1}$ and $u_{2}$ is adjacent to $v_{2}$ in $G_{2}$ or $u_{1}$ is adjacent to $v_{1}$ in $G_{1}$ and $u_{2}=v_{2}$ [23].

Theorem 5. For $m, n \geq 1$ and $r \geq 2$, we have

$$
\tau\left(K_{r} \times K_{m, n}\right)=r^{r-2} m^{n-1} n^{m-1}(m+r)^{(r-1)(n-1)}
$$

We can generalize the above lemma as follows.

$$
\times(n+r)^{(r-1)(m-1)}(m+n+r)^{r-1} \text {. }
$$


Mathematical Problems in Engineering

3

Proof. Applying Lemma 1, we have

$$
\begin{aligned}
& \tau\left(K_{r} \times K_{m, n}\right) \\
& =\frac{1}{(r(m+n))^{2}} \operatorname{det}(r(m+n) I-\bar{D}+\bar{A}) \\
& =\frac{1}{r^{2}(m+n)^{2}}
\end{aligned}
$$

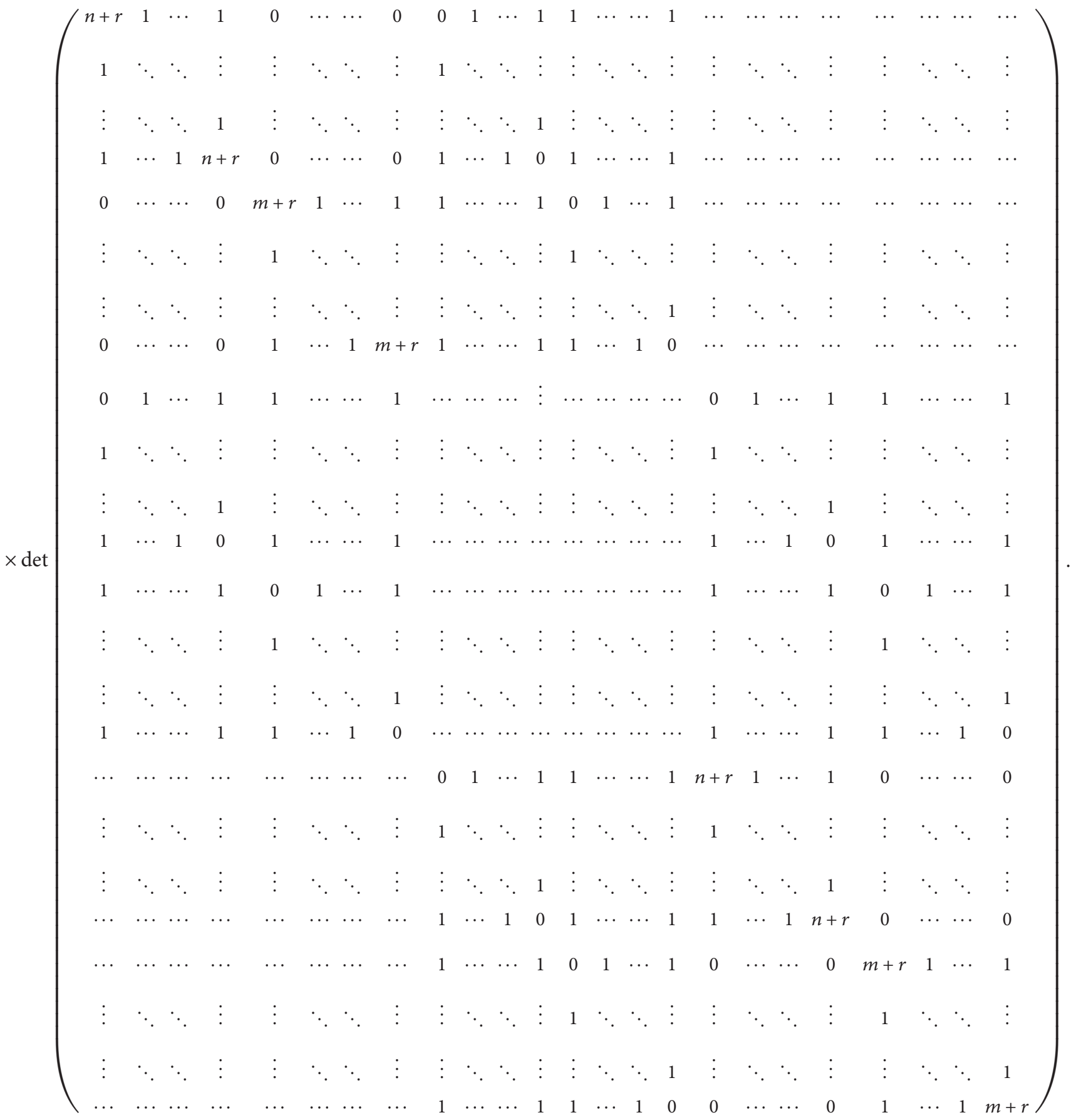

(9) 
Using Lemma 3, we get

$$
\begin{aligned}
& \tau\left(K_{r} \times K_{m, n}\right)=\frac{1}{r^{2}(m+n)^{2}} \operatorname{det}\left(\begin{array}{cccccc}
A & B & \cdots & \cdots & \cdots & B \\
B & A & \ddots & \ddots & \ddots & \vdots \\
\vdots & \ddots & \ddots & \ddots & \ddots & \vdots \\
\vdots & \ddots & \ddots & \ddots & \ddots & \vdots \\
\vdots & \ddots & \ddots & \ddots & A & B \\
B & \cdots & \cdots & \cdots & B & A
\end{array}\right) \\
& =\frac{1}{(r(m+n))^{2}}[\operatorname{det}(A-B)]^{r-1}[\operatorname{det}(A+(r-1) B)] \\
& =\frac{1}{r^{2}(m+n)^{2}} \\
& \times\left(\operatorname{det}\left(\begin{array}{cccccccc}
n+r & 0 & \cdots & 0 & -1 & \cdots & \cdots & -1 \\
0 & \ddots & \ddots & \vdots & \vdots & \ddots & \ddots & \vdots \\
\vdots & \ddots & \ddots & 0 & \vdots & \ddots & \ddots & \vdots \\
0 & \cdots & 0 & n+r & -1 & \cdots & \cdots & -1 \\
-1 & \cdots & \cdots & -1 & m+r & 0 & \cdots & 0 \\
\vdots & \ddots & \ddots & \vdots & 0 & \ddots & \ddots & \vdots \\
\vdots & \ddots & \ddots & \vdots & \vdots & \ddots & \ddots & 0 \\
-1 & \cdots & \cdots & -1 & 0 & \cdots & 0 & m+r
\end{array}\right)\right)^{r-1} \\
& \times \operatorname{det}\left(\begin{array}{cccccccc}
n+r & r & \cdots & r & (r-1) & \cdots & \cdots & (r-1) \\
r & \ddots & \ddots & \vdots & \vdots & \ddots & \ddots & \vdots \\
\vdots & \ddots & \ddots & r & \vdots & \ddots & \ddots & \vdots \\
r & \cdots & r & n+r & (r-1) & \cdots & \cdots & (r-1) \\
(r-1) & \cdots & \cdots & (r-1) & m+r & r & \cdots & r \\
\vdots & \ddots & \ddots & \vdots & r & \ddots & \ddots & \vdots \\
\vdots & \ddots & \ddots & \vdots & \vdots & \ddots & \ddots & r \\
(r-1) & \cdots & \cdots & (r-1) & r & \cdots & r & m+r
\end{array}\right) \\
& =\frac{1}{r^{2}(m+n)^{2}}\left(\operatorname{det}\left(\begin{array}{cc}
A & B \\
B^{T} & C
\end{array}\right)\right)^{r-1} \times \operatorname{det}\left(\begin{array}{cc}
D & E \\
E^{T} & F
\end{array}\right) .
\end{aligned}
$$


Using Lemma 4, we obtain

$$
\begin{aligned}
& \tau\left(K_{r} \times K_{m, n}\right)=\frac{1}{r^{2}(m+n)^{2}} \times(\operatorname{det} A)^{r-1}\left(\operatorname{det}\left(C-B^{T} A^{-1} B\right)\right)^{r-1} \times \operatorname{det} D \operatorname{det}\left(F-E^{T} D^{-1} E\right) \\
& =\frac{1}{r^{2}(m+n)^{2}}\left(\operatorname{det}\left(\begin{array}{cccc}
n+r & 0 & \cdots & 0 \\
0 & \ddots & \ddots & \vdots \\
\vdots & \ddots & \ddots & 0 \\
0 & \cdots & 0 & n+r
\end{array}\right)_{m \times m}\right)^{r-1} \\
& \times\left(\operatorname{det}\left(\begin{array}{cccc}
\frac{n(m+r)+(r-1) m+r^{2}}{n+r} & \frac{-m}{n+r} & \cdots & \frac{-m}{n+r} \\
\frac{-m}{n+r} & \ddots & \ddots & \vdots \\
\vdots & \ddots & \ddots & \frac{-m}{n+r} \\
\frac{-m}{n+r} & \cdots & \frac{-m}{n+r} & \frac{n(m+r)+(r-1) m+r^{2}}{n+r}
\end{array}\right)_{n \times n}^{n-1}\right)^{r-1} \\
& \times \operatorname{det}\left(\begin{array}{cccc}
n+r & r & \cdots & r \\
r & \ddots & \ddots & \vdots \\
\vdots & \ddots & \ddots & r \\
r & \cdots & r & n+r
\end{array}\right)_{m \times m} \\
& \times \operatorname{det}\left(\begin{array}{cccc}
\frac{n(m+r)+r m^{2}+(2 r-1) m}{n+r m} & \frac{r n+(2 r-1) m}{n+r m} & \ldots & \frac{r n+(2 r-1) m}{n+r m} \\
\frac{r n+(2 r-1) m}{n+r m} & \ddots & \ddots & \vdots \\
\vdots & \ddots & \frac{r n+(2 r-1) m}{n+r m} & \frac{n(m+r)+r m^{2}+(2 r-1) m}{n+r m}
\end{array}\right)_{n \times n}^{\frac{r n+(2 r-1) m}{n+r m}} \\
& =\frac{1}{r^{2}(m+n)^{2}}(n+r)^{m(r-1)}\left(\frac{-m}{n+r}\right)^{n(r-1)} \\
& \times\left(\operatorname{det}\left(\begin{array}{cccc}
\frac{n(m+r)+(r-1) m+r^{2}}{-m} & 1 & \cdots & 1 \\
1 & \ddots & \ddots & \vdots \\
\vdots & \ddots & \ddots & 1 \\
1 & \cdots & 1 & \frac{n(m+r)+(r-1) m+r^{2}}{-m}
\end{array}\right)_{n \times n}\right)^{r-1}
\end{aligned}
$$




$$
\times r^{m} \operatorname{det}\left(\begin{array}{cccc}
\frac{n+r}{r} & 1 & \cdots & 1 \\
1 & \ddots & \ddots & \vdots \\
\vdots & \ddots & \ddots & 1 \\
1 & \cdots & 1 & \frac{n+r}{r}
\end{array}\right)_{m \times m} \times\left(\frac{r n+(2 r-1) m}{n+r m}\right)^{n}
$$$$
\times \operatorname{det}\left(\begin{array}{cccc}
\frac{n(m+r)+r m^{2}+(2 r-1) m}{r n+(2 r-1) m} & 1 & \cdots & 1 \\
1 & \ddots & \ddots & \vdots \\
\vdots & \ddots & \ddots & 1 \\
1 & \cdots & 1 & \frac{n(m+r)+r m^{2}+(2 r-1) m}{r n+(2 r-1) m}
\end{array}\right)_{n \times n}
$$

Using Lemma 2, we have

$$
\begin{aligned}
\tau & \left(K_{r} \times K_{m, n}\right) \\
= & \frac{1}{r^{2}(m+n)^{2}} \times(n+r)^{m(r-1)} \\
& \times\left(\frac{-m}{n+r}\right)^{n(r-1)}\left[-\frac{n m+r n+(r-1) m+r^{2}}{m}+n-1\right]^{r-1} \\
& \times\left[-\frac{n m+r n+(r-1) m+r^{2}}{m}-1\right]^{(r-1)(n-1)} \\
& \times r^{m}\left(\frac{n+r}{r}+m-1\right)\left(\frac{n+r}{r}-1\right)^{m-1} \\
& \times(n+r m) \times n^{m-1} \times \frac{1}{(n+r m)^{n}} \times r(m+n)^{2} \\
& \times\left[\frac{n r+(2 r-1) m}{n+r m}\right) \\
& \times\left[\frac{n(m+r)+r m^{2}+(2 r-1) m}{r n+(2 r-1) m}-1\right]^{(n-1)} \\
& \times\left[\frac{n(m+r)+r m^{2}+(2 r-1) m}{r n+(2 r-1) m}+n-1\right]
\end{aligned}
$$

$$
\begin{aligned}
& \times(n+r m)^{n-1} \times m^{n-1} \\
= & r^{r-2} \times(n+r)^{(m-n)(r-1)} \times m^{n-1} \times n^{m-1} \\
& \times(m+n+r)^{r-1} \times\left(r m+r n+m n+r^{2}\right)^{(r-1)(n-1)} \\
= & r^{r-2} \times m^{n-1} \times n^{m-1} \times(m+r)^{(r-1)(n-1)} \\
& \times(n+r)^{(r-1)(m-1)} \times(m+n+r)^{r-1} .
\end{aligned}
$$

Specially,

$$
\begin{aligned}
\tau\left(K_{r} \times K_{n, n}\right)= & r^{r-2} \times n^{2 n-2} \times(2 n+r)^{r-1} \\
& \times(n+r)^{2(r-1)(n-1)} ; \quad n \geq 1 .
\end{aligned}
$$

\section{Number of Spanning Trees of Normal Product of Graphs}

The normal product, or the strong product, $G_{1} \circ G_{2}$, of two graphs $G_{1}$ and $G_{2}$ is the simple graph with $V\left(G_{1} \circ G_{2}\right)=V_{1} \times$ $V_{2}$, where $\left(u_{1}, u_{2}\right)$ and $\left(v_{1}, v_{2}\right)$ are adjacent in $G_{1} \circ G_{2}$ if and only if either $u_{1}=v_{1}$ and $u_{2}$ is adjacent to $v_{2}$, or $u_{1}$ is adjacent to $v_{1}$ and $u_{2}=v_{2}$, or $u_{1}$ is adjacent to $v_{1}$ and $u_{2}$ is adjacent to $v_{2},[24]$.

Theorem 6. For $m, n \geq 1$ and $r \geq 2$, we have

$$
\begin{aligned}
\tau\left(K_{r} \circ K_{m, n}\right)= & r^{r m+r n-2} m^{n-1} n^{m-1} \\
& \times(m+1)^{n(r-1)}(n+1)^{m(r-1)} .
\end{aligned}
$$


Proof. Applying Lemma 1, we have

$$
\begin{aligned}
& \tau\left(K_{r} \circ K_{m, n}\right) \\
& =\frac{1}{(r(m+n))^{2}} \operatorname{det}(r(m+n) I-\bar{D}+\bar{A}) \\
& =\frac{1}{r^{2}(m+n)^{2}}
\end{aligned}
$$

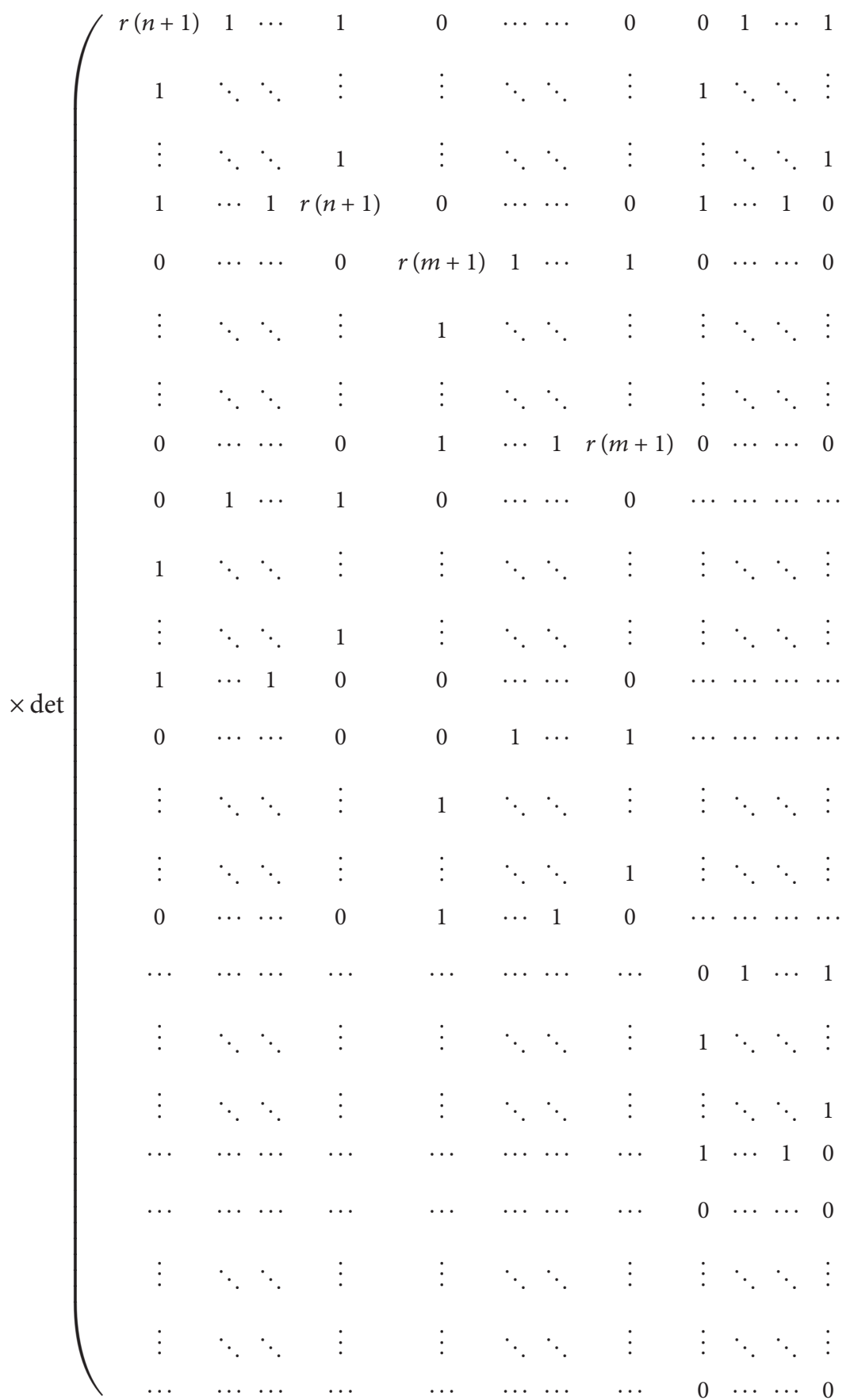




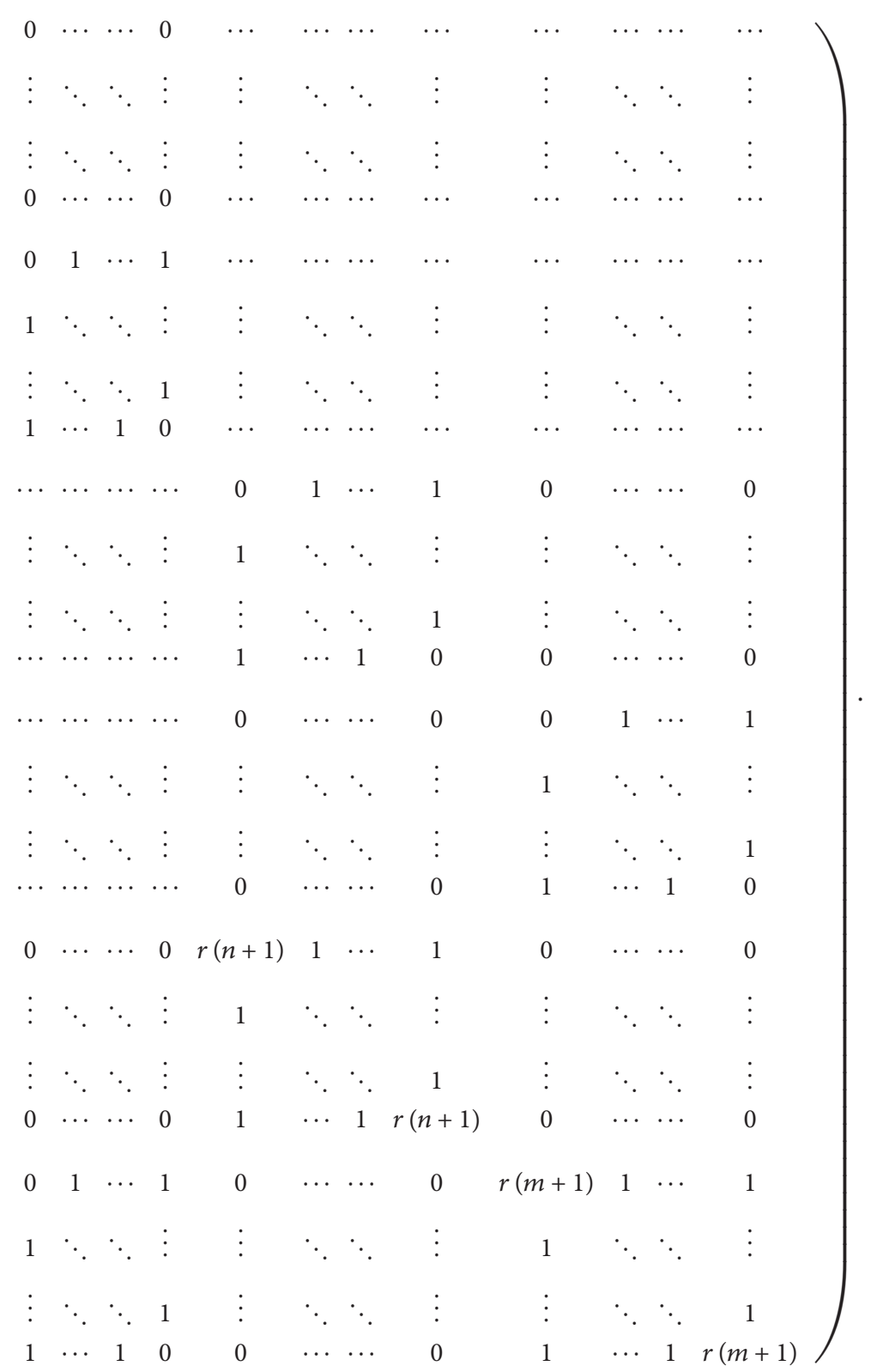

Using Lemma 3, we get

$$
\tau\left(K_{r} \circ K_{m, n}\right) \quad\left(\begin{array}{cccccc}
A & B & \cdots & \cdots & \cdots & B \\
B & A & \ddots & \ddots & \ddots & \vdots \\
\vdots & \ddots & \ddots & \ddots & \ddots & \vdots \\
\vdots & \ddots & \ddots & \ddots & \ddots & \vdots \\
\vdots & \ddots & \ddots & \ddots & A & B \\
B & \cdots & \cdots & \cdots & B & A
\end{array}\right)
$$




$$
\begin{aligned}
& =\frac{1}{(r(m+n))^{2}}[\operatorname{det}(A-B)]^{r-1}[\operatorname{det}(A+(r-1) B)] \\
& =\frac{1}{r^{2}(m+n)^{2}}\left(\operatorname{det}\left(\begin{array}{cccccccc}
r(n+1) & 0 & \cdots & 0 & 0 & \cdots & \cdots & 0 \\
0 & \ddots & \ddots & \vdots & \vdots & \ddots & \ddots & \vdots \\
\vdots & \ddots & \ddots & 0 & \vdots & \ddots & \ddots & \vdots \\
0 & \cdots & 0 & r(n+1) & 0 & \cdots & \cdots & 0 \\
0 & \cdots & \cdots & 0 & r(m+1) & 0 & \cdots & 0 \\
\vdots & \ddots & \ddots & \vdots & 0 & \ddots & \ddots & \vdots \\
\vdots & \ddots & \ddots & \vdots & \vdots & \ddots & \ddots & 0 \\
0 & \cdots & \cdots & 0 & 0 & \cdots & 0 & r(m+1)
\end{array}\right)\right)^{r-1} \\
& \times \operatorname{det}\left(\begin{array}{cccccccc}
r(n+1) & r & \cdots & r & 0 & \cdots & \cdots & 0 \\
r & \ddots & \ddots & \vdots & \vdots & \ddots & \ddots & \vdots \\
\vdots & \ddots & \ddots & r & \vdots & \ddots & \ddots & \vdots \\
r & \cdots & r & r(n+1) & 0 & \cdots & \cdots & 0 \\
0 & \cdots & \cdots & 0 & r(m+1) & r & \cdots & r \\
\vdots & \ddots & \ddots & \vdots & r & \ddots & \ddots & \vdots \\
\vdots & \ddots & \ddots & \vdots & \vdots & \ddots & \ddots & r \\
0 & \cdots & \cdots & 0 & r & \cdots & r & r(m+1)
\end{array}\right) \\
& =\frac{1}{r^{2}(m+n)^{2}}\left(\operatorname{det}\left(\begin{array}{cccc}
r(n+1) & 0 & \cdots & 0 \\
0 & \ddots & \ddots & \vdots \\
\vdots & \ddots & \ddots & 0 \\
0 & \cdots & 0 & r(n+1)
\end{array}\right)_{m \times m}\right)^{r-1} \\
& \times\left(\operatorname{det}\left(\begin{array}{cccc}
r(m+1) & 0 & \cdots & 0 \\
0 & \ddots & \ddots & \vdots \\
\vdots & \ddots & \ddots & 0 \\
0 & \cdots & 0 & r(m+1)
\end{array}\right)_{n \times n}\right)^{r-1} \\
& \times \operatorname{det}\left(\begin{array}{cccc}
r(n+1) & r & \cdots & r \\
r & \ddots & \ddots & \vdots \\
\vdots & \ddots & \ddots & r \\
r & \cdots & r & r(n+1)
\end{array}\right) \times \operatorname{det}\left(\begin{array}{cccc}
r(m+1) & r & \cdots & r \\
r & \ddots & \ddots & \vdots \\
\vdots & \ddots & \ddots & r \\
r & \cdots & r & r(m+1)
\end{array}\right) .
\end{aligned}
$$

Using Lemma 2, we obtain

$$
\begin{aligned}
\tau\left(K_{r} \circ K_{m, n}\right) \\
=\frac{1}{r^{2}(m+n)^{2}}(r(n+1))^{m(r-1)}(r(m+1))^{n(r-1)} \\
\quad \times\left(r^{m} \times(n+m) \times n^{m-1}\right)\left(r^{n} \times(n+m) \times m^{n-1}\right) \\
=r^{r(m+n)-2} m^{n-1} n^{m-1}(m+1)^{n(r-1)}(n+1)^{m(r-1)} .
\end{aligned}
$$

Specially,

$$
\tau\left(K_{r} \circ K_{n, n}\right)=r^{2(r n-1)} \times n^{2(n-1)} \times(n+1)^{2 n(r-1)} ; \quad n \geq 1 .
$$

\section{Number of Spanning Trees of Composition Product of Graphs}

The composition, or lexicographic product, $G_{1}\left[G_{2}\right]$, of two graphs $G_{1}$ and $G_{2}$ is the simple graph with $V_{1} \times V_{2}$ as the vertex set in which the vertices $\left(u_{1}, u_{2}\right)$ and $\left(v_{1}, v_{2}\right)$ are adjacent if either $u_{1}$ is adjacent to $v_{1}$ or $u_{1}=v_{1}$ and $u_{2}$ is adjacent to $v_{2}$ in $G_{2}$ [24]. 
Theorem 7. For $m, n \geq 1$ and $r \geq 2$, we get

$$
\times(r m+r n-n)^{r(n-1)}
$$

$$
\tau\left(K_{r}\left[K_{m, n}\right]\right)=r^{2(r-1)}(m+n)^{2(r-1)}(r m+r n-m)^{r(m-1)} \quad \text { Proof. Applying Lemma 1, we have }
$$

$$
\begin{gathered}
\tau\left(K_{r}\left[K_{m, n}\right]\right) \\
=\frac{1}{r^{2}(m+n)^{2}}
\end{gathered}
$$

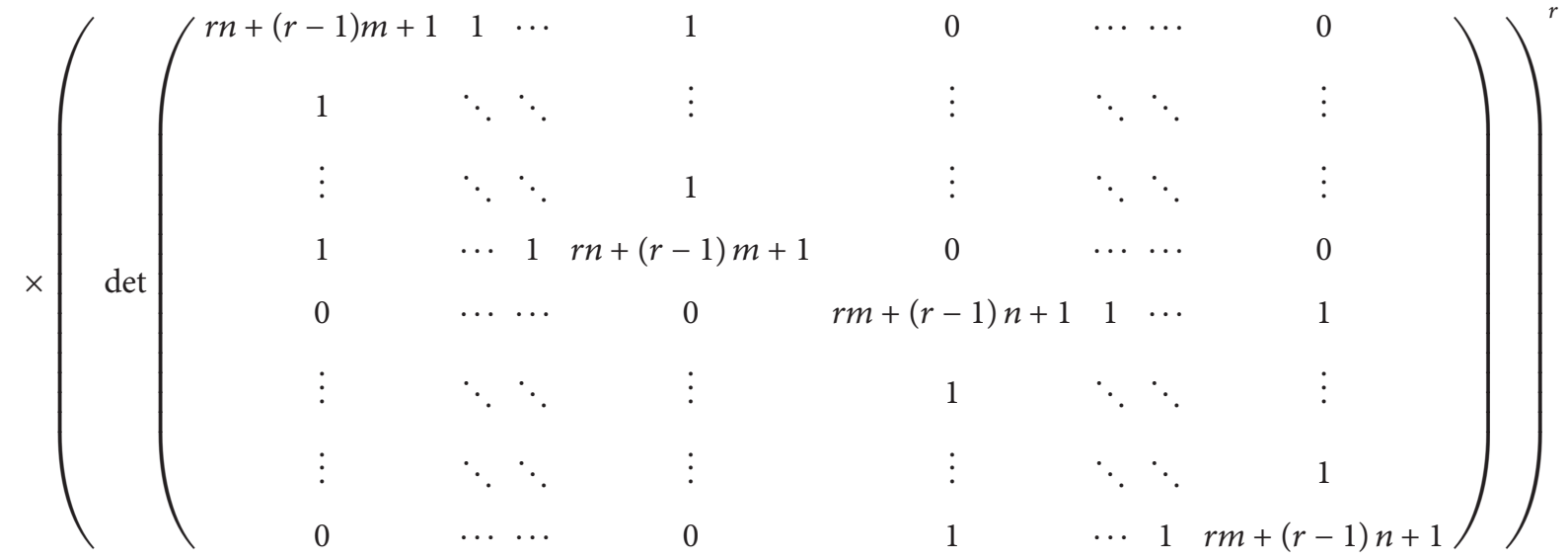

$$
=\frac{1}{r^{2}(m+n)^{2}}\left(\operatorname{det}\left(\begin{array}{cccc}
r n+(r-1) m+1 & 1 & \cdots & 1 \\
1 & \ddots & \ddots & \vdots \\
\vdots & \ddots & \ddots & 1 \\
1 & \cdots & 1 & r n+(r-1) m+1
\end{array}\right)_{m \times m}\right)^{r}
$$

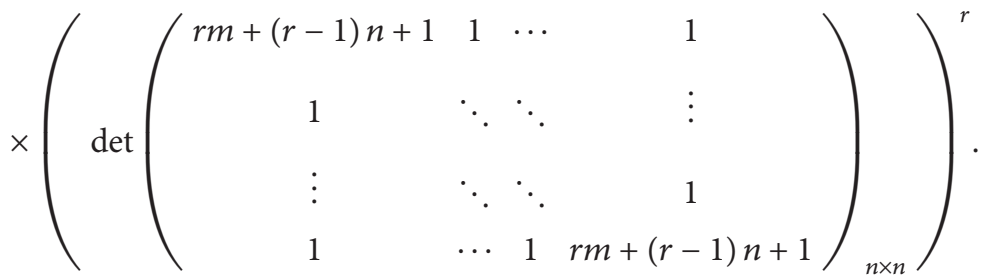

Using Lemma 2, we obtain

$$
\begin{aligned}
\tau\left(K_{r}\left[K_{m, n}\right]\right)= & r^{2(r-1)}(m+n)^{2(r-1)} \\
& \times(r m+r n-m)^{r(m-1)}(r m+r n-n)^{r(n-1)} .
\end{aligned}
$$

Specially,

$$
\tau\left(K_{r}\left[K_{n, n}\right]\right)=(2 r)^{2(r-1)} n^{2(r n-1)}(2 r-1)^{2 r(n-1)} ; \quad n \geq 1 .
$$

\section{Complexity of Tensor Product of Graphs}

The tensor product, or Kronecker product, $G_{1} \otimes G_{2}$, of two graphs $G_{1}$ and $G_{2}$ is the simple graph with $V\left(G_{1} \otimes G_{2}\right)=V_{1} \times$ $V_{2}$, where $\left(u_{1}, u_{2}\right)$ and $\left(v_{1}, v_{2}\right)$ are adjacent in $G_{1} \otimes G_{2}$ if and only if $u_{1}$ is adjacent to $v_{1}$ in $G_{1}$ and $u_{2}$ is adjacent to $v_{2}$ in $G_{2}$ [24].

Theorem 8. For $m, n \geq 1$ and $r \geq 2$, we have

$$
\tau\left(K_{r} \otimes K_{m, n}\right)=r^{r-2}(r-2)^{r-1}(r-1)^{r(m+n-2)+1} m^{r n-1} n^{r m-1} .
$$


Proof. Applying Lemma 1, we get

$$
\begin{aligned}
& \tau\left(K_{r} \otimes K_{m, n}\right) \\
& =\frac{1}{(r(m+n))^{2}} \operatorname{det}(r(m+n) I-\bar{D}+\bar{A}) \\
& =\frac{1}{r^{2}(m+n)^{2}}
\end{aligned}
$$

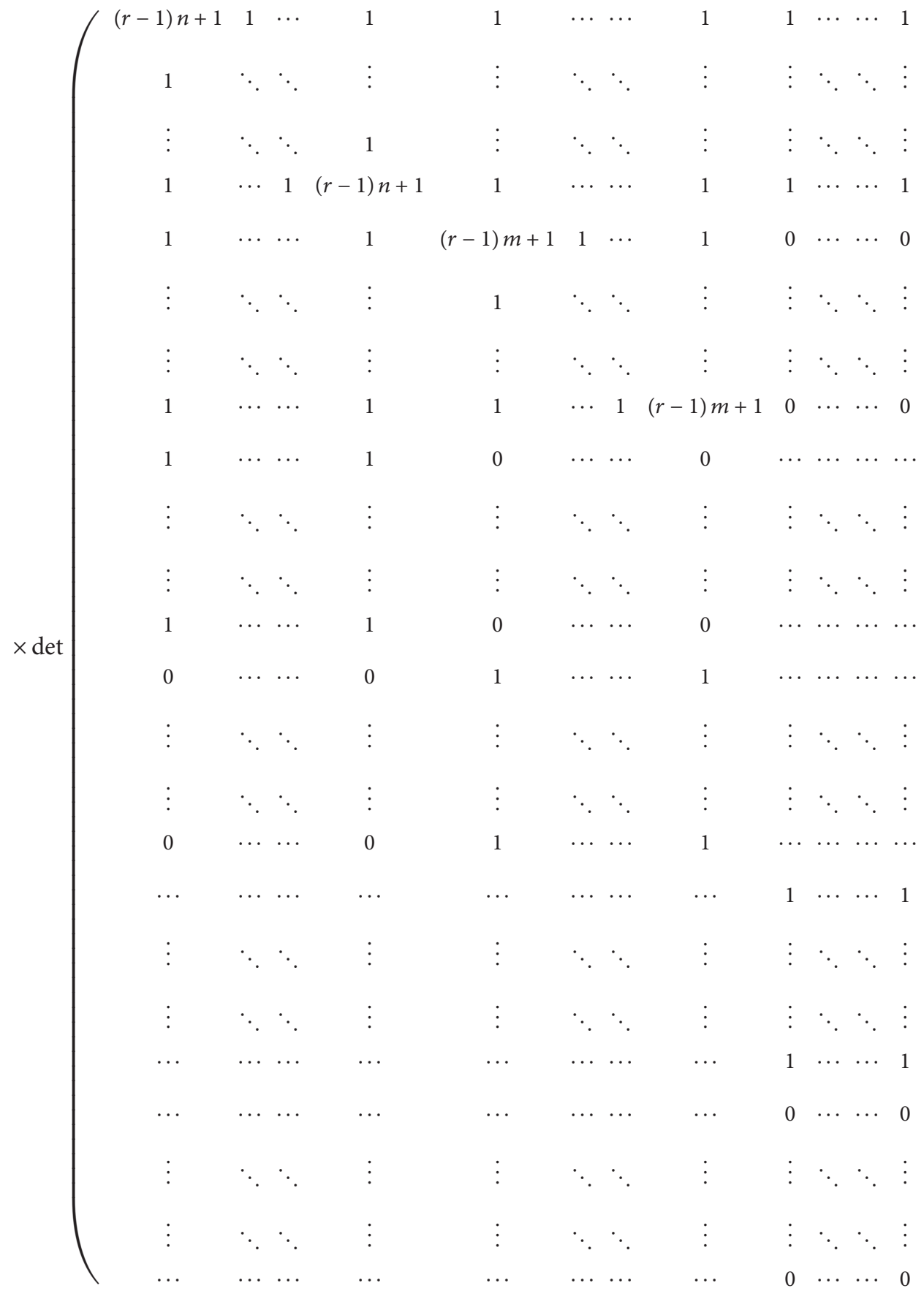




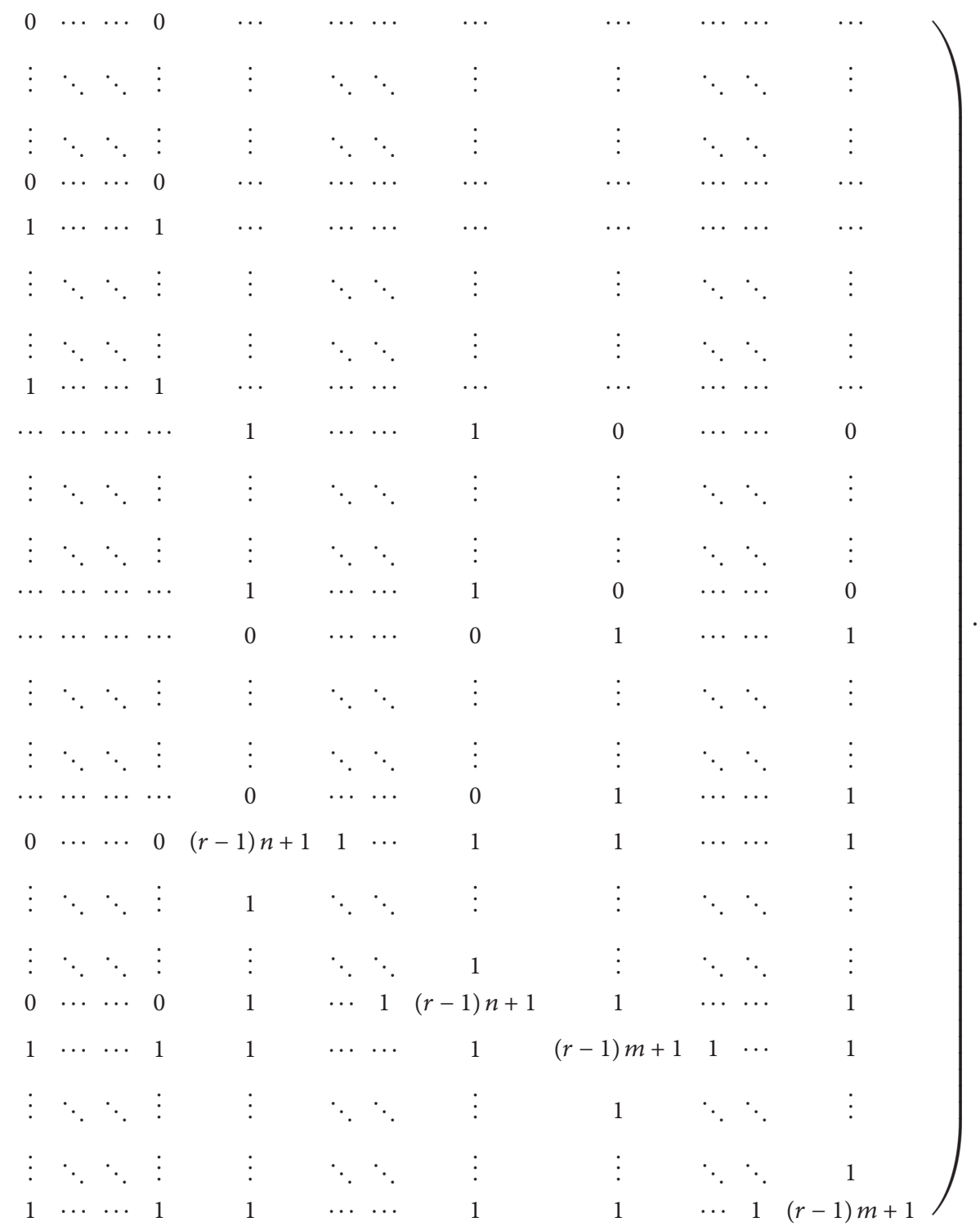

Using Lemma 3, we obtain

$$
=\frac{1}{r^{2}(m+n)^{2}} \operatorname{det}\left(\begin{array}{cccccc}
A & B & \cdots & \cdots & \cdots & B \\
B & A & \ddots & \ddots & \ddots & \vdots \\
\vdots & \ddots & \ddots & \ddots & \ddots & \vdots \\
\vdots & \ddots & \ddots & \ddots & \ddots & \vdots \\
\vdots & \ddots & \ddots & \ddots & A & B \\
B & \cdots & \cdots & \cdots & B & A
\end{array}\right)
$$




$$
\begin{aligned}
& =\frac{1}{(r(m+n))^{2}}[\operatorname{det}(A-B)]^{r-1}[\operatorname{det}(A+(r-1) B)] \\
& =\frac{1}{r^{2}(m+n)^{2}} \\
& \times\left(\operatorname{det}\left(\begin{array}{cccccccc}
(r-1) n & 0 & \cdots & 0 & 1 & \cdots & \cdots & 1 \\
0 & \ddots & \ddots & \vdots & \vdots & \ddots & \ddots & \vdots \\
\vdots & \ddots & \ddots & 0 & \vdots & \ddots & \ddots & \vdots \\
0 & \cdots & 0 & (r-1) n & 1 & \cdots & \cdots & 1 \\
1 & \cdots & \cdots & 1 & (r-1) m & 0 & \cdots & 0 \\
\vdots & \ddots & \ddots & \vdots & 0 & \ddots & \ddots & \vdots \\
\vdots & \ddots & \ddots & \vdots & \vdots & \ddots & \ddots & 0 \\
1 & \cdots & \cdots & 1 & 0 & \cdots & 0 & (r-1) m
\end{array}\right)^{r-1}\right. \\
& \times \operatorname{det}\left(\begin{array}{cccccccc}
(r-1) n+r & r & \cdots & r & 1 & \cdots & 1 \\
r & \ddots & \ddots & \vdots & \vdots & \ddots & \ddots & \vdots \\
\vdots & \ddots & \ddots & r & \vdots & \ddots & \ddots & \vdots \\
r & \cdots & r & (r-1) n+r & 1 & \cdots & \cdots & 1 \\
1 & \cdots & \cdots & 1 & (r-1) m+r & r & \cdots & r \\
\vdots & \ddots & \ddots & \vdots & r & \ddots & \ddots & \vdots \\
\vdots & \ddots & \ddots & \vdots & \vdots & \ddots & \ddots & r \\
1 & \cdots & \cdots & 1 & r & \cdots & r & (r-1) m+r
\end{array}\right) \\
& =\frac{1}{r^{2}(m+n)^{2}}\left(\operatorname{det}\left(\begin{array}{cc}
A & B \\
B^{T} & C
\end{array}\right)\right)^{r-1} \times \operatorname{det}\left(\begin{array}{cc}
D & E \\
E^{T} & F
\end{array}\right) .
\end{aligned}
$$

\section{Using Lemma 4, we obtain}

$$
\begin{aligned}
& \tau\left(K_{r} \otimes K_{m, n}\right) \\
& =\frac{1}{r^{2}(m+n)^{2}} \times(\operatorname{det} A)^{r-1}\left(\operatorname{det}\left(C-B^{T} A^{-1} B\right)\right)^{r-1} \times \operatorname{det} D \operatorname{det}\left(F-E^{T} D^{-1} E\right) \\
& \left.=\frac{1}{r^{2}(m+n)^{2}}\left(\operatorname{det}\left(\begin{array}{cccc}
(r-1) n & 0 & \cdots & 0 \\
0 & \ddots & \ddots & \vdots \\
\vdots & \ddots & \ddots & 0 \\
0 & \cdots & 0 & (r-1) n
\end{array}\right)_{m \times m}\right)^{r-1}\right)^{{ }^{2}}
\end{aligned}
$$


14

Mathematical Problems in Engineering

$$
\begin{aligned}
& \times \operatorname{det}\left(\begin{array}{cc}
\frac{\left[(r-1)^{2} m+\left(r^{2}-r\right)\right] n+\left(r^{2}-r\right) m^{2}+\left(r^{2}-1\right) m}{r m+(r-1) n} & \frac{\left(r^{2}-1\right) m+\left(r^{2}-r\right) n}{r m+(r-1) n} \\
\frac{\left(r^{2}-1\right) m+\left(r^{2}-r\right) n}{r m+(r-1) n} & \ddots \\
\vdots & \ddots \\
\frac{\left(r^{2}-1\right) m+\left(r^{2}-r\right) n}{r m+(r-1) n} & \cdots
\end{array}\right. \\
& \ldots \quad \frac{\left(r^{2}-1\right) m+\left(r^{2}-r\right) n}{r m+(r-1) n} \\
& \ddots \quad \vdots \\
& \ddots \quad \frac{\left(r^{2}-1\right) m+\left(r^{2}-r\right) n}{r m+(r-1) n} \\
& \left.\frac{\left(r^{2}-1\right) m+\left(r^{2}-r\right) n}{r m+(r-1) n} \frac{\left[(r-1)^{2} m+\left(r^{2}-r\right)\right] n+\left(r^{2}-r\right) m^{2}+\left(r^{2}-1\right) m}{r m+(r-1) n}\right)_{n \times n} \\
& \times\left(\operatorname{det}\left(\begin{array}{cccc}
\frac{m\left[(r-1)^{2} n-1\right]}{(r-1) n} & \frac{-m}{(r-1) n} & \cdots & \frac{-m}{(r-1) n} \\
\frac{-m}{(r-1) n} & \ddots & \ddots & \vdots \\
\vdots & \ddots & \ddots & \frac{-m}{(r-1) n} \\
\frac{-m}{(r-1) n} & \cdots & \frac{-m}{(r-1) n} & \frac{m\left[(r-1)^{2} n-1\right]}{(r-1) n}
\end{array}\right)_{n \times n}\right)^{r-1} \\
& \times \operatorname{det}\left(\begin{array}{cccc}
(r-1) n+r & r & \cdots & r \\
r & \ddots & \ddots & \vdots \\
\vdots & \ddots & \ddots & r \\
r & \cdots & r & (r-1) n+r
\end{array}\right)_{m \times m} \\
& =\frac{1}{r^{2}(m+n)^{2}}((r-1) n)^{m(r-1)} \\
& \left.\times\left(\frac{-m}{(r-1) n}\right)^{n(r-1)} \operatorname{det}\left(\begin{array}{cccc}
-\left[(r-1)^{2} n-1\right] & 1 & \cdots & 1 \\
1 & \ddots & \ddots & \vdots \\
\vdots & \ddots & \ddots & 1 \\
1 & \cdots & 1 & -\left[(r-1)^{2} n-1\right]
\end{array}\right)_{n \times n}\right)^{r-1}
\end{aligned}
$$


$\times r^{m} \operatorname{det}\left(\begin{array}{cccc}\frac{(r-1) n+r}{r} & 1 & \cdots & 1 \\ 1 & \ddots & \ddots & \vdots \\ \vdots & \ddots & \ddots & 1 \\ 1 & \cdots & 1 & \frac{(r-1) n+r}{r}\end{array}\right)_{m \times m} \times\left(\frac{\left(r^{2}-1\right) m+\left(r^{2}-r\right) n}{r m+(r-1) n}\right)^{n}$

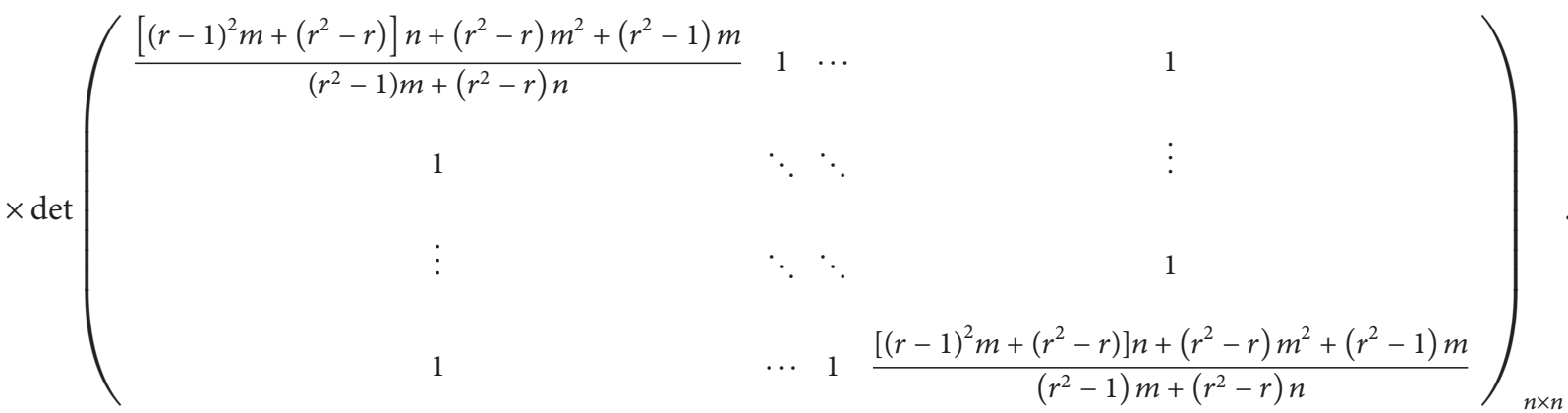

Using Lemma 2 yields

$$
\begin{aligned}
& \tau\left(K_{r} \otimes K_{m, n}\right) \\
&=\frac{1}{r^{2}(m+n)^{2}} \times(r-1)^{(r-1)(m-n)} \\
& \quad \times n^{(r-1)(m-n)} \times m^{n(r-1)} \times[r n(r-2)]^{r-1} \\
& \quad \times\left[n(r-1)^{2}\right]^{(r-1)(n-1)} \times[r m+(r-1) n] \\
& \quad \times[(r-1) n]^{m-1} \times \frac{1}{(r m+(r-1) n)^{n}} \\
& \quad \times\left[r(r-1)(m+n)^{2}\right] \\
& \quad \times[m(r-1)(r m+(r-1) n)]^{n-1} \\
&= \times m^{r-2} \times(r-1)^{r(m+n)-2 r+1} \times(r-2)^{r-1} \\
& \\
& \\
& \\
& \\
& \\
& \\
&
\end{aligned}
$$

Specially,

$$
\begin{aligned}
& \tau\left(K_{r} \otimes K_{n, n}\right) \\
& \quad=r^{r-2} \times(r-2)^{r-1} \times(r-1)^{2 r(n-1)+1} \times n^{2(n r-1)} ; \quad n \geq 1 .
\end{aligned}
$$

\section{Number of Spanning Trees of Symmetric Product of Graphs}

The symmetric product, $G_{1} \oplus G_{2}$, of two graphs $G_{1}$ and $G_{2}$ is the simple graph with $V\left(G_{1} \oplus G_{2}\right)=V_{1} \times V_{2}$, where $\left(u_{1}, u_{2}\right)$ and $\left(v_{1}, v_{2}\right)$ are adjacent in $G_{1} \oplus G_{2}$ if and only if either $u_{1}$ is adjacent to $v_{1}$ in $G_{1}$ and $u_{2}$ is not adjacent to $v_{2}$ in $G_{2}$ or $u_{1}$ is not adjacent to $v_{1}$ in $G_{1}$ and $u_{2}$ is adjacent to $v_{2}$ in $G_{2}$ [24].

Theorem 9. For $m, n \geq 1$ and $r \geq 2$, we have

$$
\begin{aligned}
& \tau\left(K_{r} \oplus K_{m, n}\right) \\
& =r^{r-2} \times((r-1) m+n)^{r(m-1)} \\
& \quad \times((r-1) n+m)^{r(n-1)} \times\left(m^{2}+n^{2}+r m n\right)^{r-1} .
\end{aligned}
$$


Proof. Applying Lemma 1, we have

$$
\begin{aligned}
& \tau\left(K_{r} \oplus K_{m, n}\right) \\
& =\frac{1}{(r(m+n))^{2}} \operatorname{det}(r(m+n) I-\bar{D}+\bar{A}) \\
& =\frac{1}{r^{2}(m+n)^{2}}
\end{aligned}
$$

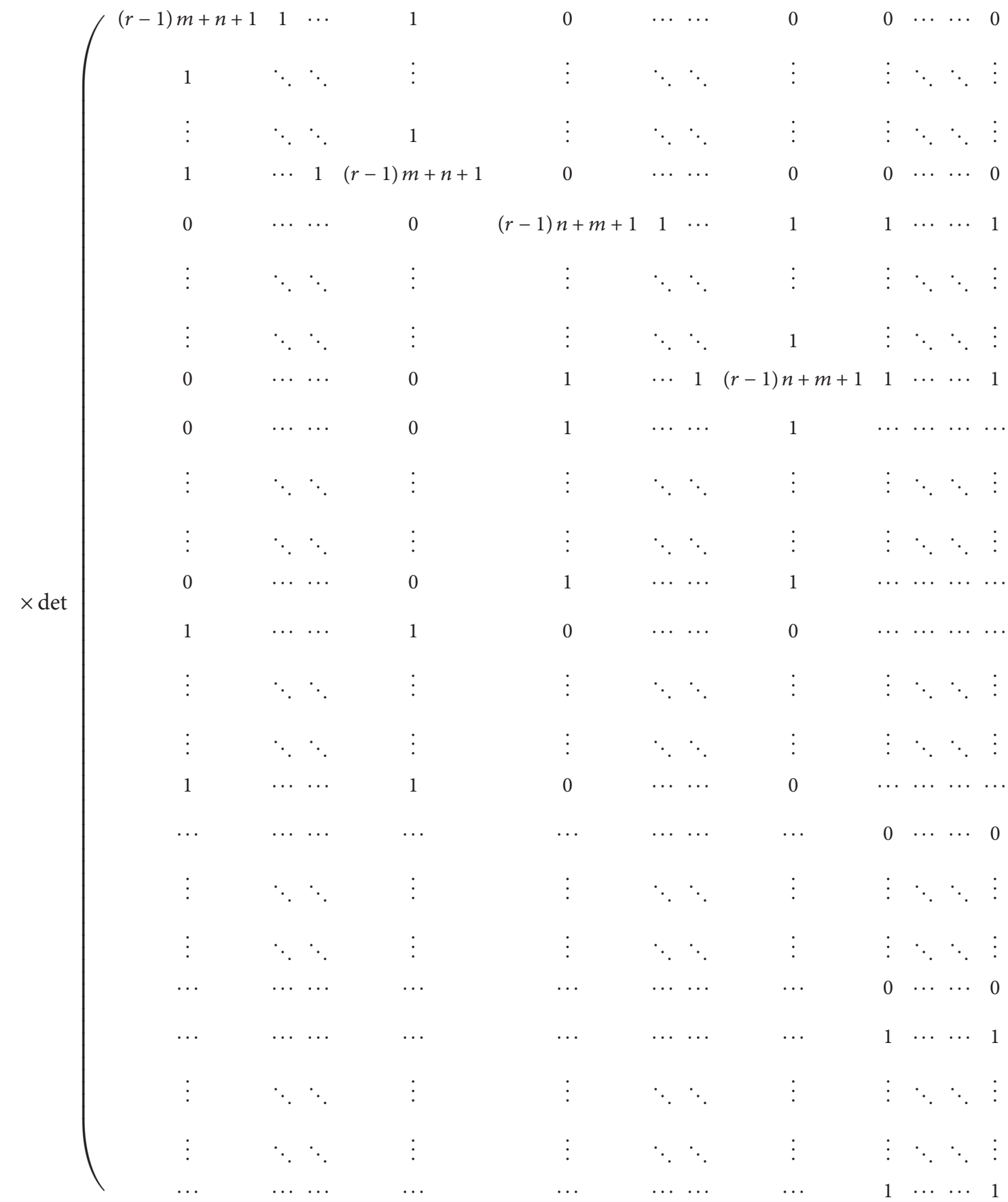




\begin{tabular}{|c|c|c|c|c|c|c|c|c|}
\hline 1 & $\begin{array}{lll}\ldots & \ldots\end{array}$ & 1 & $\cdots$ & $\cdots \quad \cdots$ & $\cdots$ & $\cdots$ & $\cdots \quad \cdots$ & $\cdots$ \\
\hline$\vdots$ & $\ddots \quad \ddots$ & $\vdots$ & $\vdots$ & $\ddots \quad \ddots$ & $\vdots$ & $\vdots$ & $\ddots \quad \ddots$ & $\vdots$ \\
\hline : & $\ddots \quad \ddots$ & $\vdots$ & $\vdots$ & $\ddots \quad \ddots$ & $\vdots$ & $\vdots$ & $\ddots \quad \ddots$ & $\vdots$ \\
\hline 1 & $\ldots \quad \ldots$ & 1 & $\ldots$ & $\ldots \ldots$ & $\ldots$ & $\ldots$ & $\ldots \quad \ldots$ & $\ldots$ \\
\hline 0 & $\ldots \quad \ldots$ & 0 & $\ldots$ & $\cdots \cdots$ & $\ldots$ & $\cdots$ & $\ldots \quad \ldots$ & $\ldots$ \\
\hline : & $\ddots \quad \ddots$ & $\vdots$ & $\vdots$ & $\ddots \quad \ddots$ & $\vdots$ & $\vdots$ & $\ddots \quad \ddots$ & $\vdots$ \\
\hline$\vdots$ & $\ddots \quad \ddots$ & $\vdots$ & $\vdots$ & $\ddots \quad \ddots$ & $\vdots$ & $\vdots$ & $\ddots \quad \ddots$ & : \\
\hline 0 & $\cdots \quad \cdots$ & 0 & $\cdots$ & $\cdots \cdots$ & $\cdots$ & $\ldots$ & $\cdots \quad \cdots$ & $\cdots$ \\
\hline .. & $\cdots \quad \cdots$ & $\cdots$ & 0 & $\cdots \cdots$ & 0 & 1 & $\ldots \ldots$ & 1 \\
\hline$\vdots$ & $\ddots$ & $\vdots$ & $\vdots$ & $\ddots \quad \ddots$ & $\vdots$ & $\vdots$ & $\ddots \quad \ddots$ & $\vdots$ \\
\hline$\vdots$ & $\ddots \quad \ddots$ & $\vdots$ & $\vdots$ & $\ddots \quad \ddots$ & $\vdots$ & $\vdots$ & $\ddots \quad \ddots$ & $\vdots$ \\
\hline$\cdots$ & $\ldots \quad \ldots$ & $\cdots$ & 0 & $\cdots \cdots$ & 0 & 1 & $\ldots \quad \ldots$ & 1 \\
\hline$\cdots$ & $\ldots \quad \ldots$ & $\cdots$ & 1 & $\cdots \quad \ldots$ & 1 & 0 & $\ldots \ldots$ & 0 \\
\hline$\vdots$ & $\ddots$ & $\vdots$ & $\vdots$ & $\ddots \quad \ddots$ & $\vdots$ & $\vdots$ & $\ddots \quad \ddots$ & $\vdots$ \\
\hline$\vdots$ & $\ddots$ & $\cdots$ & $\vdots$ & $\ddots \quad \ddots$ & $\vdots$ & $\vdots$ & $\ddots \quad \ddots$ & $\vdots$ \\
\hline$\cdots$ & $\cdots \quad \cdots$ & $\vdots$ & 1 & $\cdots \quad \cdots$ & 1 & 0 & $\begin{array}{lll} & \ldots & \ldots\end{array}$ & 0 \\
\hline 1 & $\cdots \quad \cdots$ & 1 & $(r-1) m+n+1$ & $1 \quad \cdots$ & 1 & 0 & $\begin{array}{lll}\ldots & \ldots\end{array}$ & 0 \\
\hline$\vdots$ & $\ddots$ & $\vdots$ & 1 & $\ddots \quad \ddots$ & $\vdots$ & $\vdots$ & $\ddots \quad \ddots$ & $\vdots$ \\
\hline$\vdots$ & & $\vdots$ & $\vdots$ & $\ddots \quad \ddots$ & 1 & $\vdots$ & $\ddots \quad \ddots$ & $\vdots$ \\
\hline 1 & $\cdots \quad \cdots$ & 1 & 1 & $\cdots \quad 1$ & $(r-1) m+n+1$ & 0 & $\begin{array}{lll}\cdots & \cdots\end{array}$ & 0 \\
\hline 0 & $\cdots \quad \cdots$ & 0 & 0 & $\cdots \quad \cdots$ & 0 & $(r-1) n+m+1$ & $1 \quad \cdots$ & 1 \\
\hline$\vdots$ & $\ddots \quad \ddots$ & $\vdots$ & $\vdots$ & $\ddots \quad \ddots$ & $\vdots$ & 1 & $\ddots$ & $\vdots$ \\
\hline$\vdots$ & $\ddots \quad \ddots$ & $\vdots$ & $\vdots$ & $\ddots \quad \ddots$ & $\vdots$ & $\vdots$ & $\ddots \quad \ddots$ & 1 \\
\hline 0 & $\begin{array}{lll}\ldots & \cdots\end{array}$ & 0 & 0 & $\cdots \cdots$ & 0 & 1 & $\cdots \quad 1$ & $(r-1) n+m+1$ \\
\hline
\end{tabular}

Using Lemma 3, we obtain

$$
\begin{aligned}
& \tau\left(K_{r} \oplus K_{m, n}\right) \\
& =\frac{1}{r^{2}(m+n)^{2}} \operatorname{det}\left(\begin{array}{cccccc}
A & B & \cdots & \cdots & \cdots & B \\
B & A & \ddots & \ddots & \ddots & \vdots \\
\vdots & \ddots & \ddots & \ddots & \ddots & \vdots \\
\vdots & \ddots & \ddots & \ddots & \ddots & \vdots \\
\vdots & \ddots & \ddots & \ddots & A & B \\
B & \cdots & \cdots & \cdots & B & A
\end{array}\right)
\end{aligned}
$$




$$
\begin{aligned}
& =\frac{1}{(r(m+n))^{2}}[\operatorname{det}(A-B)]^{r-1}[\operatorname{det}(A+(r-1) B)] \\
& =\frac{1}{r^{2}(m+n)^{2}}
\end{aligned}
$$

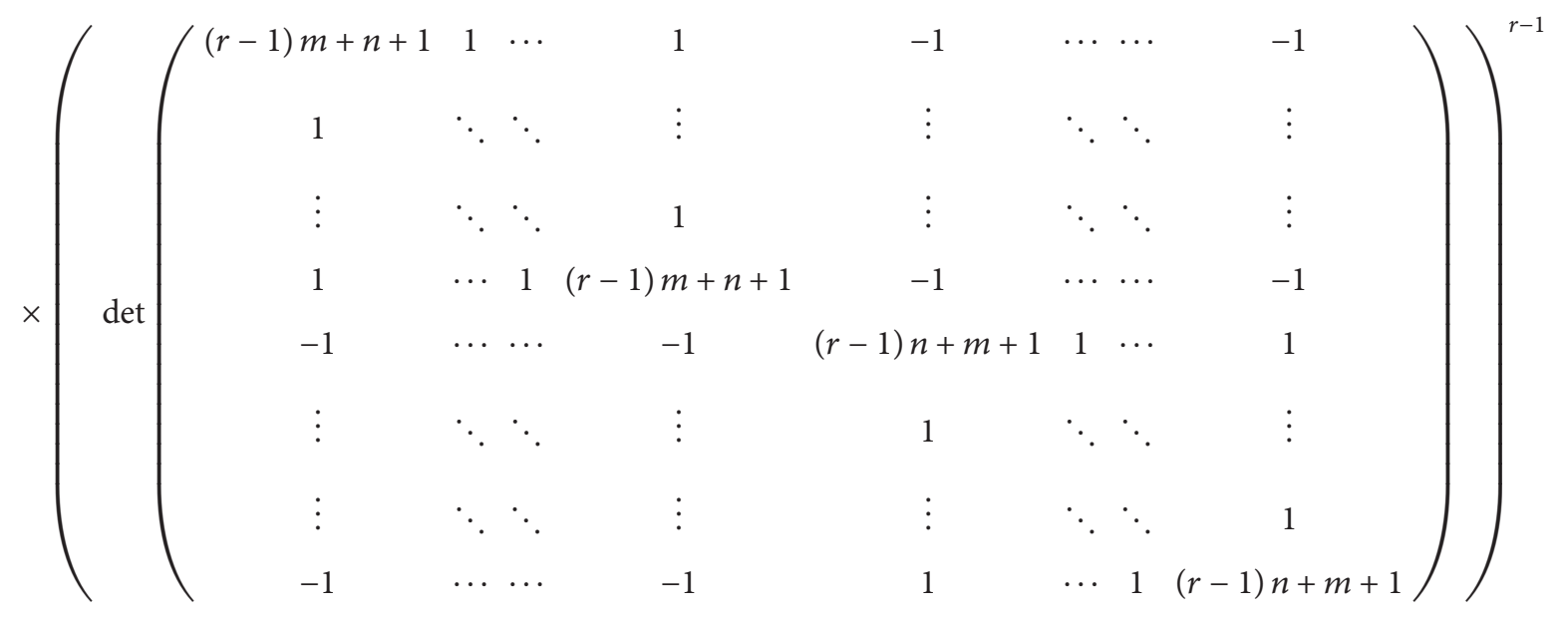

$$
\begin{aligned}
& \times \operatorname{det}\left(\begin{array}{cccccccc}
(r-1) m+n+1 & 1 & \cdots & 1 & (r-1) & \cdots & \cdots & (r-1) \\
1 & \ddots & \ddots & \vdots & \vdots & \ddots & \ddots & \vdots \\
\vdots & \ddots & \ddots & 1 & \vdots & \ddots & \ddots & \vdots \\
1 & \cdots & 1 & (r-1) m+n+1 & (r-1) & \cdots & \cdots & (r-1) \\
(r-1) & \cdots & \cdots & (r-1) & (r-1) n+m+1 & 1 & \cdots & 1 \\
\vdots & \ddots & \ddots & \vdots & 1 & \ddots & \ddots & \vdots \\
(r-1) & \cdots & \cdots & (r-1) & \vdots & \ddots & \ddots & 1 \\
& \ddots & \ddots & \cdots & 1 & (r-1) n+m+1
\end{array}\right) \\
& =\frac{1}{r^{2}(m+n)^{2}}\left(\operatorname{det}\left(\begin{array}{cc}
A & B \\
B^{T} & C
\end{array}\right)\right)^{r-1} \times \operatorname{det}\left(\begin{array}{cc}
D & E \\
E^{T} & F
\end{array}\right) .
\end{aligned}
$$

\section{Using Lemma 4, we get}

$$
\begin{aligned}
& \tau\left(K_{r} \oplus K_{m, n}\right) \\
& =\frac{1}{r^{2}(m+n)^{2}} \times(\operatorname{det} A)^{r-1}\left(\operatorname{det}\left(C-B^{T} A^{-1} B\right)\right)^{r-1} \times \operatorname{det} D \operatorname{det}\left(F-E^{T} D^{-1} E\right) \\
& =\frac{(r m+n)^{r-1}((r-1) m+n)^{(r-1)(m-1)}}{r^{2}(n+m)^{2}}
\end{aligned}
$$



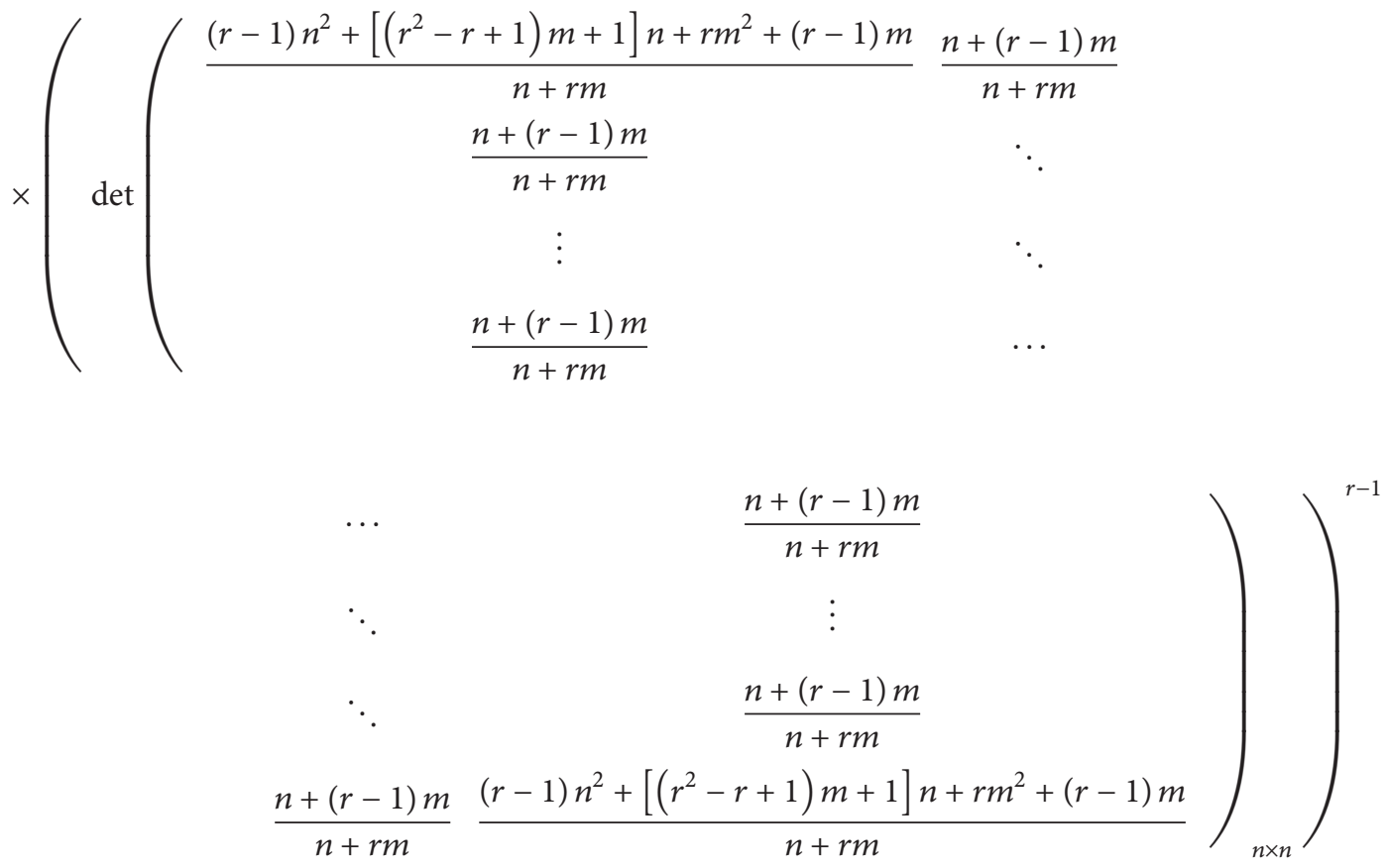

$\times(r m+n)((r-1) m+n)^{m-1}$

$\times \operatorname{det}\left(\begin{array}{cc}\frac{(r-1) n^{2}+\left[\left(r^{2}-r+1\right) m+1\right] n+r m^{2}+\left(-r^{2}+3 r-1\right) m}{n+r m} & n+\left(-r^{2}+3 r-1\right) m \\ \frac{n+\left(-r^{2}+3 r-1\right) m}{n+r m} & \ddots \\ \vdots & \ddots\end{array}\right.$

$$
\begin{aligned}
& \frac{n+\left(-r^{2}+3 r-1\right) m}{n+r m} \\
& \ddots \quad \vdots \\
& \ddots \quad \frac{n+\left(-r^{2}+3 r-1\right) m}{n+r m} \\
& \left.\frac{n+\left(-r^{2}+3 r-1\right) m}{n+r m} \frac{(r-1) n^{2}+\left[\left(r^{2}-r+1\right) m+1\right] n+r m^{2}+\left(-r^{2}+3 r-1\right) m}{n+r m}\right) \\
& n \times n \\
& =\frac{(r m+n)^{r-1}((r-1) m+n)^{(r-1)(m-1)}}{r^{2}(n+m)^{2}} \times\left(\frac{n+(r-1) m}{n+r m}\right)^{n(r-1)}
\end{aligned}
$$




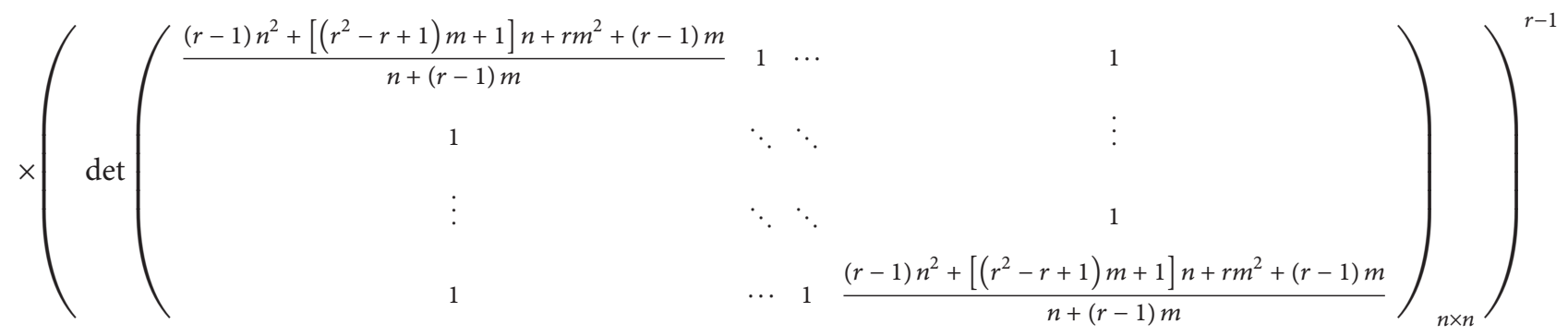

$\times(r m+n)((r-1) m+n)^{m-1} \times\left(\frac{n+\left(-r^{2}+3 r-1\right) m}{n+r m}\right)^{n}$

$\times \operatorname{det}\left(\begin{array}{c}\frac{(r-1) n^{2}+\left[\left(r^{2}-r+1\right) m+1\right] n+r m^{2}+\left(-r^{2}+3 r-1\right) m}{n+\left(-r^{2}+3 r-1\right) m} \\ 1 \\ \vdots \\ 1\end{array}\right.$

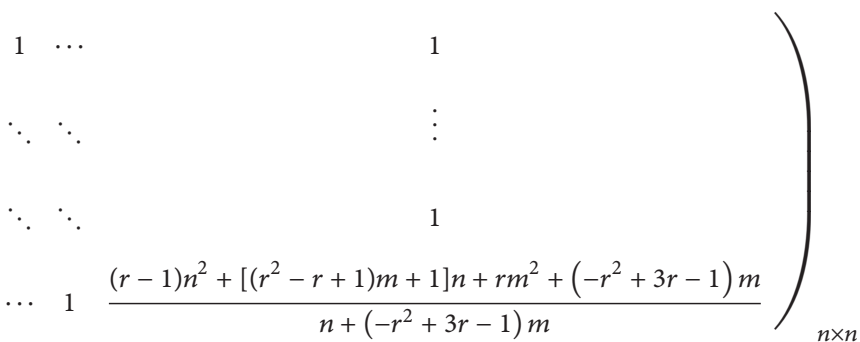

Using Lemma 2, we have

$$
\begin{aligned}
\tau & \left(K_{r} \oplus K_{m, n}\right) \\
= & \frac{1}{r^{2}(m+n)^{2}} \times(r m+n)^{r-1} \times((r-1) m+n)^{(r-1)(m-1)} \\
& \times\left(\frac{n+(r-1) m}{n+r m}\right)^{(r-1) n} \times \frac{1}{(n+(r-1) m)^{(r-1) n}} \\
& \times\left(r n^{2}+r m^{2}+r^{2} n m\right)^{r-1} \\
& \times\left(r m^{2}+\left(r^{2}-r+1\right) n m+(r-1) n^{2}\right)^{(r-1)(n-1)} \\
& \times(r m+n) \times((r-1) m+n)^{m-1} \\
& \times\left(\frac{n+\left(-r^{2}+3 r-1\right) m}{n+r m}\right)^{n} \\
& \times\left(r m^{2}+\left(r^{2}-r+1\right) n m+(r-1) n^{2}\right)^{n-1} \\
& \times \frac{1}{\left(n+\left(-r^{2}+3 r-1\right) m\right)^{n}} \times\left(r n^{2}+r m^{2}+2 r m n\right)
\end{aligned}
$$

$$
=r^{r-2} \times((r-1) m+n)^{r(m-1)}
$$$$
\times((r-1) n+m)^{r(n-1)} \times\left(m^{2}+n^{2}+r m n\right)^{r-1} .
$$

Specially,

$$
\tau\left(K_{r} \oplus K_{n, n}\right)=r^{2 r n-r-2} \times n^{2 r n-2} \times(r+2)^{r-1} ; \quad n \geq 1
$$

\section{Number of Spanning Trees of Strong Sum of Graphs}

The strong sum, $G_{1} * G_{2}$, of two graphs $G_{1}$ and $G_{2}$ is the simple graph with $V\left(G_{1} * G_{2}\right)=V_{1} \times V_{2}$ where $\left(u_{1}, u_{2}\right)$ and $\left(v_{1}, v_{2}\right)$ are adjacent in $G_{1} * G_{2}$ if and only if $u_{2}$ is adjacent to $v_{2}$ in $G_{2}$ and either $u_{1}$ is adjacent to $v_{1}$ in $G_{1}$ or $u_{1}=v_{1}$ [24].

Theorem 10. For $m, n \geq 1$ and $r \geq 2$, we have

$$
\tau\left(K_{r} * K_{m, n}\right)=r^{(m+n) r-2} \times m^{r n-1} \times n^{r m-1} .
$$


Mathematical Problems in Engineering

21

Proof. Applying Lemma 1, we have

$\tau\left(K_{r} * K_{m, n}\right)$

$=\frac{1}{(r(m+n))^{2}} \operatorname{det}(r(m+n) I-\bar{D}+\bar{A})$

$=\frac{1}{r^{2}(m+n)^{2}}$

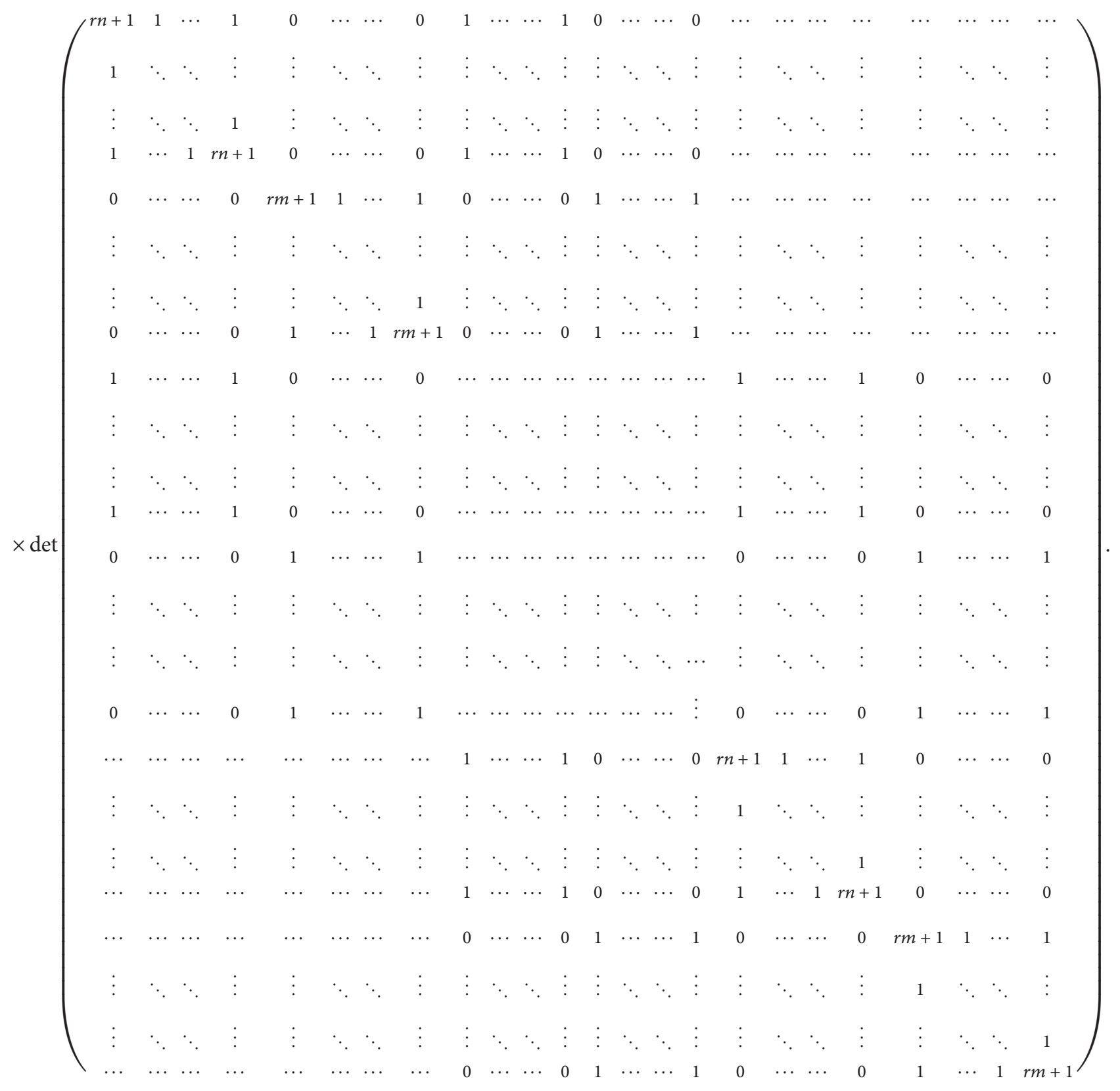

(36) 
Using Lemma 3, we obtain

$$
\begin{aligned}
& \tau\left(K_{r} * K_{m, n}\right)=\frac{1}{r^{2}(m+n)^{2}} \operatorname{det}\left(\begin{array}{cccccc}
A & B & \cdots & \cdots & \cdots & B \\
B & A & \ddots & \ddots & \ddots & \vdots \\
\vdots & \ddots & \ddots & \ddots & \ddots & \vdots \\
\vdots & \ddots & \ddots & \ddots & \ddots & \vdots \\
\vdots & \ddots & \ddots & \ddots & A & B \\
B & \cdots & \cdots & \cdots & B & A
\end{array}\right) \\
& =\frac{1}{(r(m+n))^{2}}[\operatorname{det}(A-B)]^{r-1}[\operatorname{det}(A+(r-1) B)] \\
& =\frac{1}{r^{2}(m+n)^{2}}\left(\begin{array}{cccccccc}
r n & 0 & \cdots & 0 & 0 & \cdots & \cdots & 0 \\
0 & \ddots & \ddots & \vdots & \vdots & \ddots & \ddots & \vdots \\
\vdots & \ddots & \ddots & 0 & \vdots & \ddots & \ddots & \vdots \\
0 & \cdots & 0 & r n & 0 & \cdots & \cdots & 0 \\
0 & \cdots & \cdots & 0 & r m & 0 & \cdots & 0 \\
\vdots & \ddots & \ddots & \vdots & 0 & \ddots & \ddots & \vdots \\
\vdots & \ddots & \ddots & \vdots & \vdots & \ddots & \ddots & 0 \\
0 & \cdots & \cdots & 0 & 0 & \cdots & 0 & r m
\end{array}\right)^{r-1} \\
& \times \operatorname{det}\left(\begin{array}{cccccccc}
r(n+1) & r & \cdots & r & 0 & \cdots & \cdots & 0 \\
r & \ddots & \ddots & \vdots & \vdots & \ddots & \ddots & \vdots \\
\vdots & \ddots & \ddots & r & \vdots & \ddots & \ddots & \vdots \\
r & \cdots & r & r(n+1) & 0 & \cdots & \cdots & 0 \\
0 & \cdots & \cdots & 0 & r(m+1) & r & \cdots & r \\
\vdots & \ddots & \ddots & \vdots & r & \ddots & \ddots & \vdots \\
\vdots & \ddots & \ddots & \vdots & \vdots & \ddots & \ddots & r \\
0 & \cdots & \cdots & 0 & r & \cdots & r & r(m+1)
\end{array}\right) \\
& =\frac{1}{r^{2}(m+n)^{2}}\left[(r n)^{m}(r m)^{n}\right]^{r-1} \times r^{m} \operatorname{det}\left(\begin{array}{cccc}
n+1 & 1 & \cdots & 1 \\
1 & \ddots & \ddots & \vdots \\
\vdots & \ddots & \ddots & 1 \\
1 & \cdots & 1 & n+1
\end{array}\right)_{m \times m} \\
& \times r^{n} \operatorname{det}\left(\begin{array}{cccc}
m+1 & 1 & \cdots & 1 \\
1 & \ddots & \ddots & \vdots \\
\vdots & \ddots & \ddots & 1 \\
1 & \cdots & 1 & m+1
\end{array}\right)_{n \times n}
\end{aligned}
$$


Using Lemma 2, we get

$$
\begin{aligned}
\tau\left(K_{r} * K_{m, n}\right)= & \frac{1}{r^{2}(m+n)^{2}} \times r^{(m+n)(r-1)} \\
& \times n^{m(r-1)} \times m^{n(r-1)} \times r^{(m+n)} \\
& \times(n+1+m-1)(n+1-1)^{m-1} \\
& \times(m+1+n-1)(m+1-1)^{n-1} \\
= & r^{(m+n) r-2} \times m^{n r-1} \times n^{m r-1} .
\end{aligned}
$$

Specially,

$$
\tau\left(K_{r} * K_{n, n}\right)=(n r)^{2(r n-1)} ; \quad n \geq 1
$$

\section{Conclusion}

Driving formulas for different types of graphs can prove to be helpful in identifying those graphs that contain the maximum number of spanning trees. Such an investigation has practical consequence related to network reliability. Some computationally hard problems, such as the Steiner tree problem and the traveling salesperson problem, can be solved approximately by using spanning trees [25]. Due to the high dependence of the network design and reliability on the graph theory we introduced the above important theorems and lemmas and their proofs.

\section{Conflict of Interests}

The author declares that there is no conflict of interests regarding the publication of this paper.

\section{References}

[1] C. J. Colbourn, The Combinatoric of Network Reliability, Oxford University Press, New York, NY, USA, 1987.

[2] W. Myrvold, K. H. Cheung, L. B. Page, and J. E. Perry, "Uniformly most reliable networks do not always exist," Networks, vol. 21, no. 4, pp. 417-419, 1991.

[3] L. Petingi, F. Boesch, and C. Suffel, "On the characterization of graphs with maximum number of spanning trees," Discrete Mathematics, vol. 179, no. 1-3, pp. 155-166, 1998.

[4] L. G. Valiant, "The complexity of enumeration and reliability problems," SIAM Journal on Computing, vol. 8, no. 3, pp. 410421, 1979.

[5] T. J. N. Brown, R. B. Mallion, P. Pollak, and A. Roth, "Some methods for counting the spanning trees in labelled molecular graphs, examined in relation to certain fullerenes," Discrete Applied Mathematics, vol. 67, no. 1-3, pp. 51-66, 1996.

[6] M. Mihail and P. Winkler, "On the number of Eulerian orientations of a graph," Algorithmica, vol. 16, no. 4-5, pp. 402-414, 1996.

[7] G. G. Kirchhoff, "Über die auflösung der gleichungen, auf welche man be ider Untersuchung der linearen verteilung galvanischer störme gefuhrt wird," Annalen der Physik und Chemie, vol. 72, pp. 497-508, 1847.
[8] A. Cayley, "A theorm on trees," Quarterly Journal of Mathematics, vol. 23, pp. 276-378, 1889.

[9] H. Prüfer, "Neuer beweis satzes über permutationen," Archiv für Mathematik und Physik, vol. 27, pp. 142-144, 1918.

[10] T. L. Austin, "The enumeration of point labelled chromatic graphs and trees," Canadian Journal of Mathematics, vol. 12, pp. 535-545, 1960.

[11] O. Eğecioğlu and J. B. Remmel, "Bijections for Cayley trees, spanning trees, and their $q$-analogues," Journal of Combinatorial Theory A, vol. 42, no. 1, pp. 15-30, 1986.

[12] O. Eğecioğlu and J. B. Remmel, "A bijection for spanning trees of complete multipartite graphs," Congressus Numerantium, vol. 100, pp. 225-243, 1994.

[13] R. P. Lewis, "The number of spanning trees of a complete multipartite graph," Discrete Mathematics, vol. 197-198, pp. 537-541, 1999.

[14] D. Cvetkovič, M. Doob, and H. Sach, "Spectra of graphs," in Mathematics, vol. 87, Academic Press, New York, NY, USA, 1980.

[15] T. Atajan, X. Yong, and H. Inaba, "Further analysis of the number of spanning trees in circulant graphs," Discrete Mathematics, vol. 306 , no. 22 , pp. $2817-2827,2006$.

[16] X. Chen, Q. Lin, and F. Zhang, "The number of spanning trees in odd valent circulant graphs," Discrete Mathematics, vol. 282, no. 1-3, pp. 69-79, 2004.

[17] X.-R. Yong and T. Acenjian, "The numbers of spanning trees of the cubic cycle $C_{N}^{3}$ and the quadruple cycle $C_{N}^{4}$, Discrete Mathematics, vol. 169, no. 1-3, pp. 293-298, 1997.

[18] Y. Zhang, X. Yong, and M. J. Golin, "The number of spanning trees in circulant graphs," Discrete Mathematics, vol. 223, no. 13, pp. 337-350, 2000.

[19] S. N. Daoud, "Number of spanning trees of circulant graphs $C_{6}$ and their applications," Journal of Mathematics and Statistics, vol. 8, no. 1, pp. 24-31, 2012.

[20] S. N. Daoud, "On the complexity of a class of pyramid graphs and Chebyshev polynomials," Mathematical Problems in Engineering, vol. 2013, Article ID 820549, 11 pages, 2013.

[21] S. N. Daoud, "Chebyshev polynomials and spanning tree formulas," International Journal of Mathematical Combinatorics, vol. 4, pp. 68-79, 2012.

[22] M. Marcus, A Servy of Matrix Theory and Matrix Inequalities, Allyn and Bacon, Boston, Mass, USA, 1964.

[23] J. L. Gross and T. W. Tucker, Topological Graph Theory, John Wiley \& Sons, New York, NY, USA, 1987.

[24] R. Balakrishnan and K. Ranganathan, A Textbook of Graph Theory, Springer, New York, NY, USA, 2000.

[25] D. L. Applegate, R. E. Bixby, V. Chvátal, and W. J. Cook, The Traveling Salesman Problem: A Computational Study, Princeton University Press, 2006. 


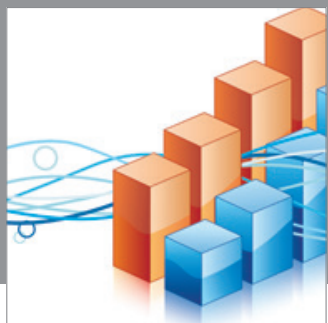

Advances in

Operations Research

mansans

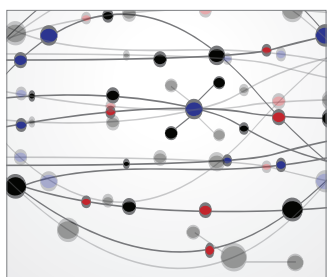

The Scientific World Journal
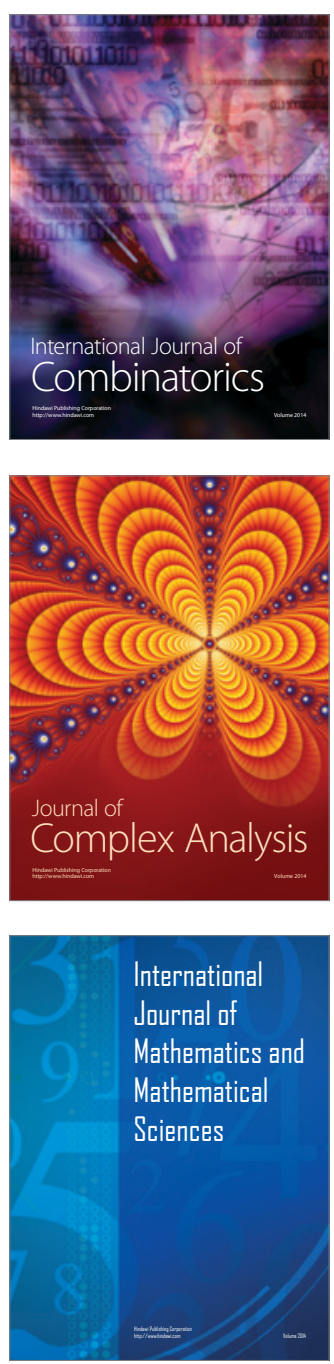
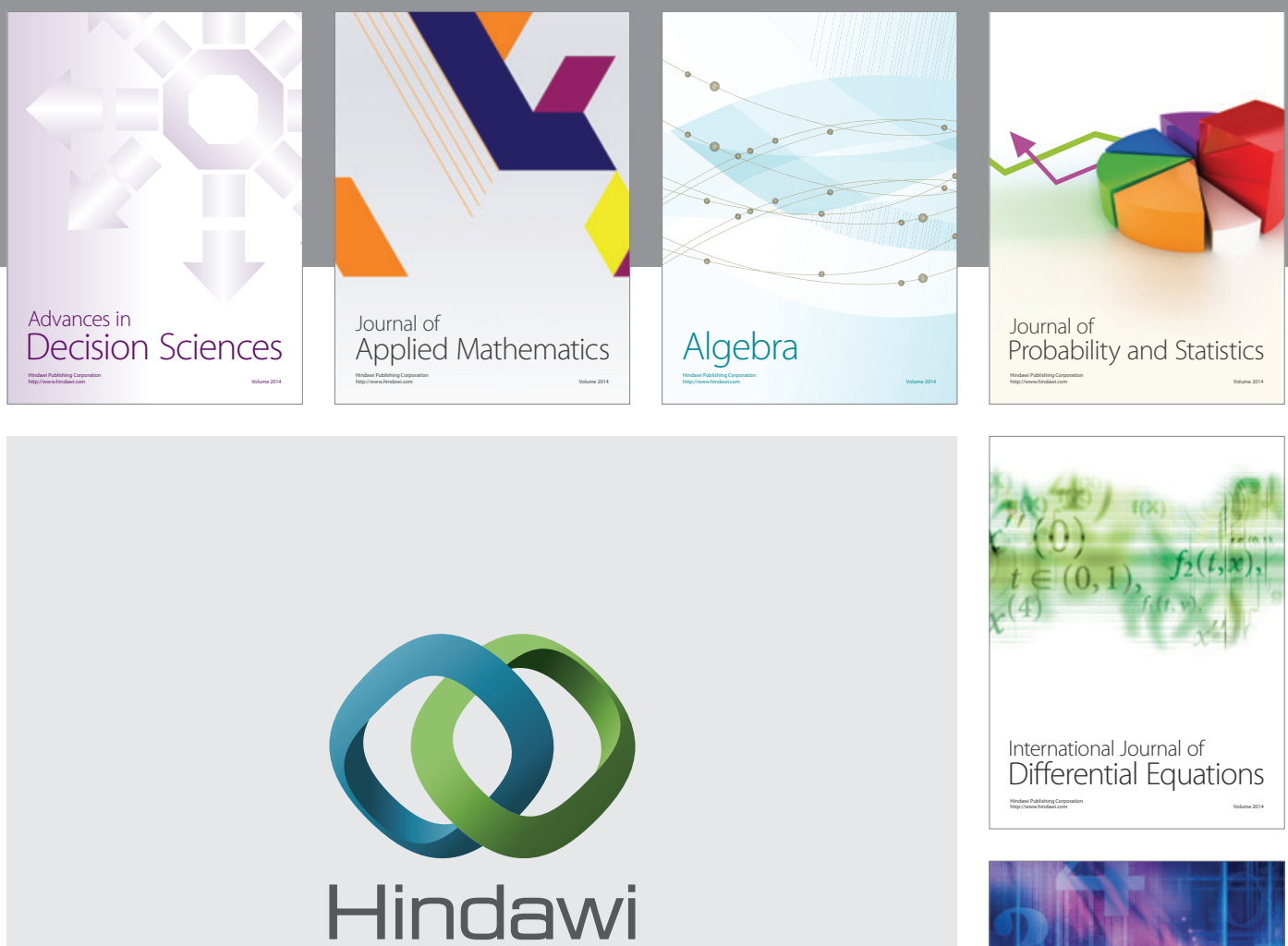

Submit your manuscripts at http://www.hindawi.com
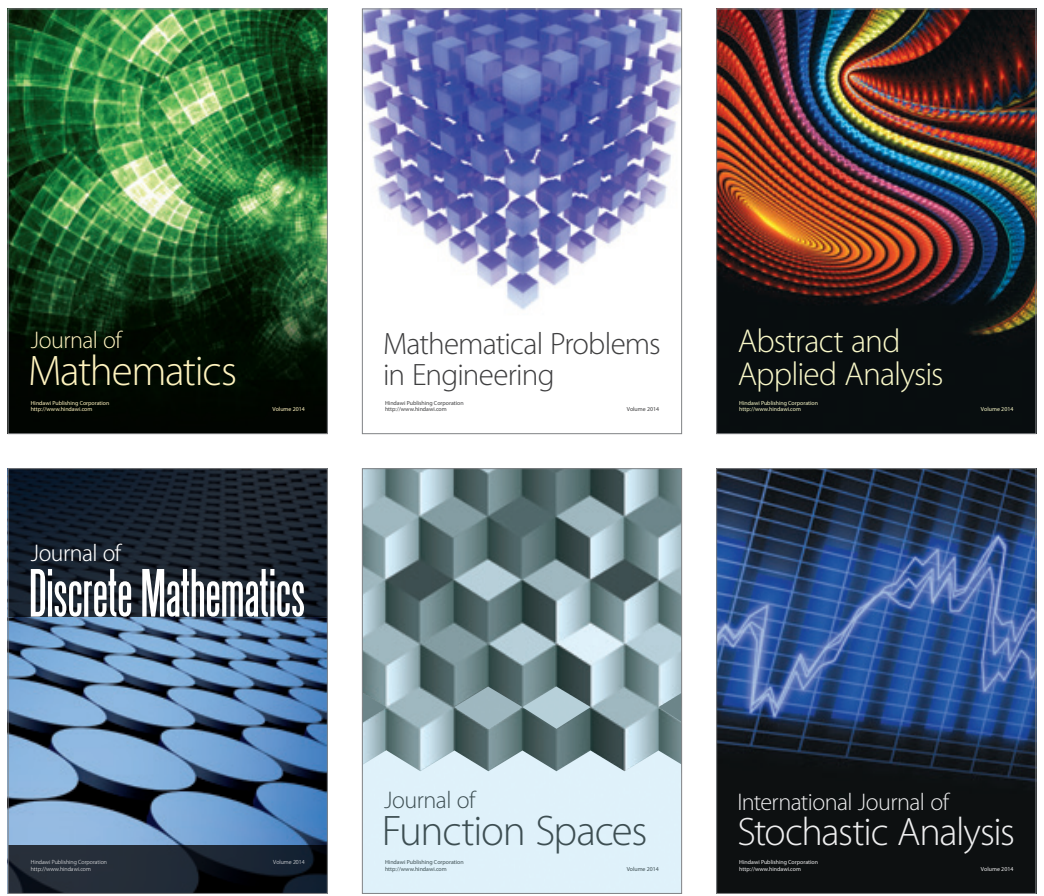

Journal of

Function Spaces

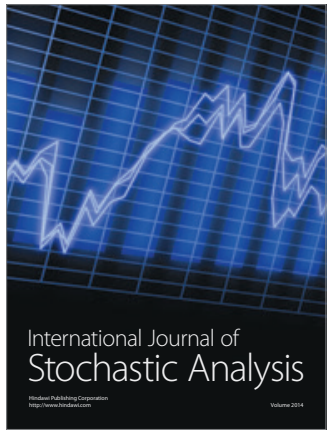

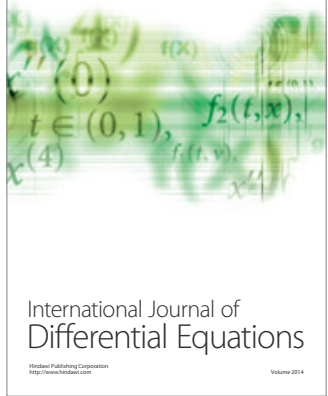
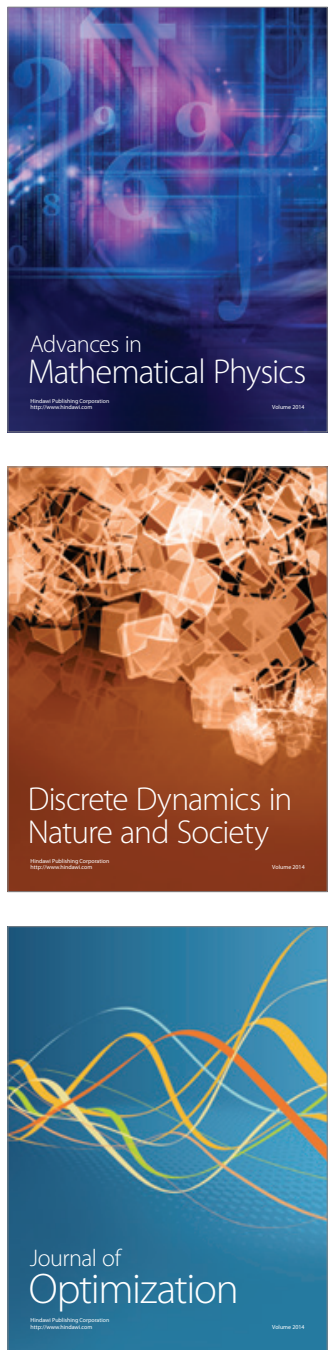